\title{
Intracellular VEGF regulates the balance between osteoblast and adipocyte differentiation
}

\author{
Yanqiu Liu, ${ }^{1}$ Agnes D. Berendsen, ${ }^{1}$ Shidong Jia, ${ }^{2}$ Sutada Lotinun, ${ }^{3}$ Roland Baron, ${ }^{3}$ \\ Napoleone Ferrara, ${ }^{4}$ and Bjorn R. Olsen ${ }^{1}$
}

\begin{abstract}
${ }^{1}$ Department of Developmental Biology, Harvard School of Dental Medicine, Boston, Massachusetts, USA. ${ }^{2}$ Department of Cancer Biology, Dana-Farber Cancer Institute, Boston, Massachusetts, USA. ${ }^{3}$ Department of Oral Medicine, Infection and Immunity, Harvard School of Dental Medicine, Boston, Massachusetts, USA. ${ }^{4}$ Genentech, South San Francisco, California, USA.
\end{abstract}

\begin{abstract}
Osteoporotic bones have reduced spongy bone mass, altered bone architecture, and increased marrow fat. Bone marrow stem cells from osteoporotic patients are more likely to differentiate into adipocytes than control cells, suggesting that adipocyte differentiation may play a role in osteoporosis. VEGF is highly expressed in osteoblastic precursor cells and is known to stimulate bone formation. Here we tested the hypothesis that VEGF is also an important regulator of cell fate, determining whether differentiation gives rise to osteoblasts or adipocytes. Mice with conditional VEGF deficiency in osteoblastic precursor cells exhibited an osteoporosis-like phenotype characterized by reduced bone mass and increased bone marrow fat. In addition, reduced VEGF expression in mesenchymal stem cells resulted in reduced osteoblast and increased adipocyte differentiation. Osteoblast differentiation was reduced when VEGF receptor 1 or 2 was knocked down but was unaffected by treatment with recombinant VEGF or neutralizing antibodies against VEGF. Our results suggested that VEGF controls differentiation in mesenchymal stem cells by regulating the transcription factors RUNX2 and PPAR $\gamma 2$ as well as through a reciprocal interaction with nuclear envelope proteins lamin A/C. Importantly, our data support a model whereby VEGF regulates differentiation through an intracrine mechanism that is distinct from the role of secreted VEGF and its receptors.
\end{abstract}

\section{Introduction}

Osteoporosis, skeletal fragility associated with reduced quantity and quality of bone, is a major problem in the aging population. During aging, the balance between bone formation and resorption shifts in favor of resorption, resulting in reduced bone mass and altered bone architecture. In vitro, bone marrow mesenchymal stem cells from patients with osteoporosis are more likely to differentiate into adipocytes than osteoblasts compared with cells isolated from patients with normal bone mass (1), and osteoporotic bones have increased accumulation of adipocytes in the trabecular bone marrow space (2-5). These studies and data from mouse models of osteoporosis have prompted questions about whether increased bone marrow fat is a consequence or the cause of decreased bone mass in osteoporosis (6).

Adipocytes and osteoblasts differentiate from a common mesenchymal precursor cell. Experimental evidence indicates that a substantial degree of plasticity exists between osteoblast and adipocyte lineages (7-10). Even fully differentiated osteoblasts derived from human mesenchymal stem cells are capable of transdifferentiating into adipocytes and chondrocytes, and vice versa (11). Under osteogenic conditions, human mesenchymal stem cells increase expression of both osteogenic and adipogenic markers. Thus, osteogenic and adipogenic differentiation may constitute a set of parallel events until relatively late during osteoblast development (12). This raises the question of whether osteoporosis is a consequence of age-depen-

Authorship note: Yanqiu Liu and Agnes D. Berendsen contributed equally to this work. Conflict of interest: The authors have declared that no conflict of interest exists. Citation for this article: J Clin Invest. 2012;122(9):3101-3113. doi:10.1172/JCI61209. dent alterations in the control of osteoblast/adipocyte fates in mesenchymal stem cells.

Unfortunately, our understanding of this control is incomplete. However, several cytokines or growth factors and associated signaling pathways, including BMP, Wnt, and polycystin pathways (13-20), are likely to play important regulatory roles. For several reasons, we hypothesized that vascular endothelial growth factor A (VEGF) may also be one of these factors. Osteoblastic precursor cells express high levels of VEGF as they migrate into the primary ossification center of developing endochondral bones in response to chemotactic factors, including $\operatorname{VEGF}(21,22)$. In addition, several studies suggest that VEGF can stimulate osteoblast differentiation (23-26). Finally, VEGF expression is notably reduced in multiple cell types, including mesenchymal stem cells, with age (27-33).

To test this hypothesis, we conditionally targeted VEGF at an early stage in mesenchymal cell differentiation. We took advantage of the fact that the transcription factor osterix (Osx) (34), required for differentiation of osteoblasts, is expressed at an early stage in the osteoblast lineage. Osx- and VEGF-expressing precursor cells, located in the perichondrium of cartilage templates of endochondral bones, give rise to the majority of osteoblasts, osteocytes, and stromal cells within the primary spongiosa (trabecular bone) (22). Postnatally, mice carrying floxed alleles of VEGF and expressing Cre recombinase under the control of the Osx promoter (OsxCre) exhibited an osteoporosis-like phenotype characterized by reduced bone density and an increased amount of bone marrow fat. In osteoblastic and adipocytic differentiation assays with cultures of bone marrow-derived stem cells, cells from mutant animals, or cells from control animals in which VEGF was knocked down in vitro, exhibited a substantial reduction in osteoblast dif- 
A

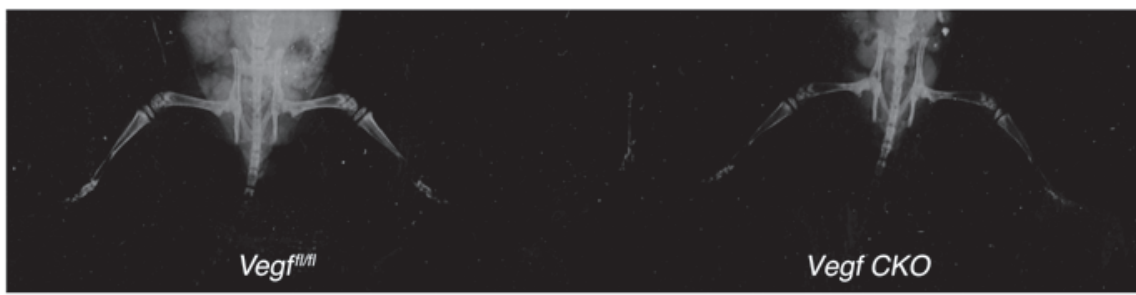

B

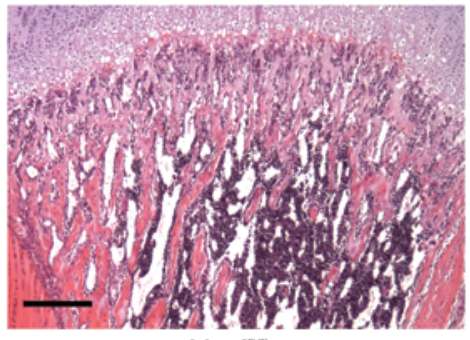

Vegf ${ }^{\text {fIIT }}$

C
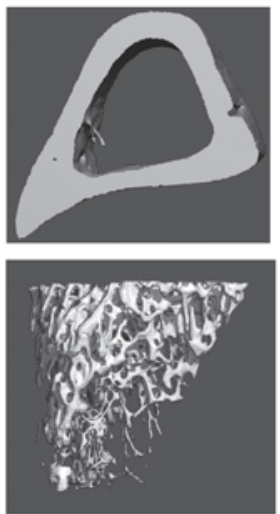

Vegf $f^{\text {hlt }}$
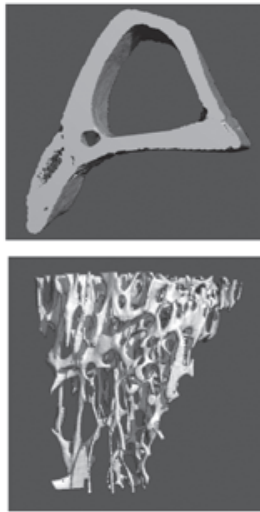

Vegf CKO

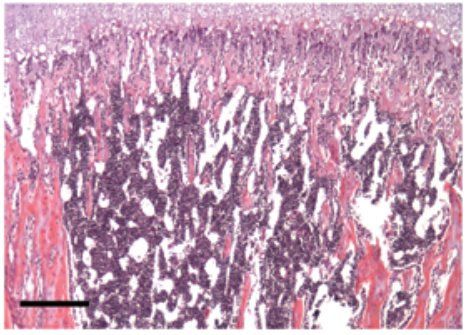

Vegf CKO

D
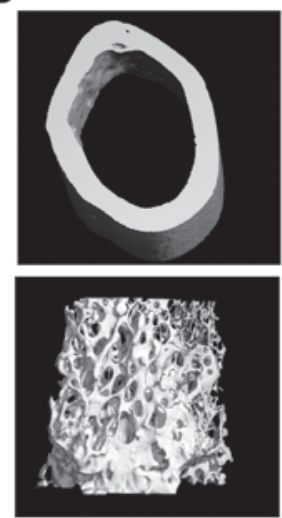

Vegfllit
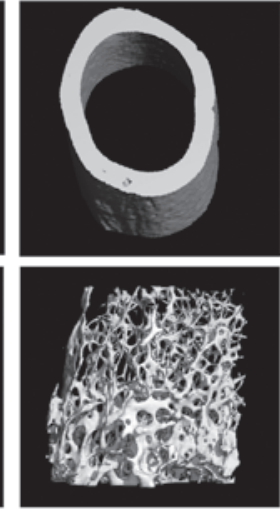

Vegf CKO

\section{Figure 1}

Reduced bone density in Vegfa CKO mice. (A) X-ray images of lower limbs of 20-day-old control (left) and Vegfa mutant (right) mice. (B) Reduced trabecular bone in sections (H\&E) from tibia of Vegfa CKO (right) compared with control (left) mice. Scale bars: $\mathbf{3 0 0} \mu \mathrm{m}$. (C) Thinner cortical bone (top) and reduced trabecular bone (bottom) in microCT images of tibia of 2-month-old mutant mice. (D) Reduced cortical bone (top) and trabecular bone (bottom) in microCT images of femur of 2-month-old mutant (right) compared with control (left) mice. ferentiation and increased adipocyte differentiation. Interestingly, addition of recombinant VEGF to mutant cell cultures or addition of neutralizing antibodies against VEGF to control cells had no effects in the assays. In contrast, retrovirus-mediated restoration of VEGF expression in mutant cells increased osteoblast differentiation and reduced adipocyte differentiation to the levels exhibited by control cells. The findings suggest that VEGF produced by osteoblastic precursor cells regulates the balance of osteoblast and adipocyte differentiation via an intracrine mechanism, distinct from mechanisms involving interactions of secreted VEGF with receptors located on the surface of target cells.

\section{Results}

Mice with reduced VEGF expression in osteoblastic lineage cells exhibit reduced bone density and increased bone marrow fat. To study the skeletal functions of osteoblast-produced VEGF, we conditionally deleted Vegfa in the osteoblast lineage by crossing mice carrying floxed Vegfa alleles (Vegfallfl) with mice expressing Cre recombinase under the control of the Osx promoter. X-ray analysis and histological examination of 3-week-old Vegfa conditional knockout (CKO) mice showed thinner femurs and reduced trabecular bone in the proximal tibia compared with control littermates (Figure 1, $\mathrm{A}$ and $\mathrm{B}$ ). These differences became more pronounced with age, and 2-month-old CKO mice showed substantially reduced bone density compared with control littermates. MicroCT analysis demonstrated a marked reduction in trabecular bone volume in mutant tibia (Figure 1C and Figure 2A) and femur (Figure 1D and Figure 2A). In femur, trabecular thickness was also reduced (data not shown). Cortical bone thickness (Figure 1, C and D) and bone volume (Figure 2, B and C) were reduced; however, since the overall bone size was smaller, cortical bone volume as percentage of total volume was not changed (Figure 2, B and C). Analysis of histological sections revealed not only decreased trabecular bone but also greatly increased adipocyte numbers in bone marrow of mutant mice (Figure 2E). Since reduced bone density could result from an imbalance between bone formation and resorption, we used tartrate-resistant acid phosphatase (TRAP) staining to assess the number of osteoclasts in bone sections. Consistent with expression of Osx-Cre in hypertrophic chondrocytes (22) and the known stimulatory effect of hypertrophic chondrocyte-generated VEGF on osteoclast recruitment into growth plate regions (35), the number of osteoclasts in the region immediately adjacent to the tibial growth plate was reduced in mutant mice compared with control littermates (Figure 2D). Thus, the reduction in bone mass was likely the result not of increased bone resorption, but rather of decreased bone formation.

Histology showed no difference in trabecular bone and bone marrow fat content between Vegfafl/fl mice and heterozygous 

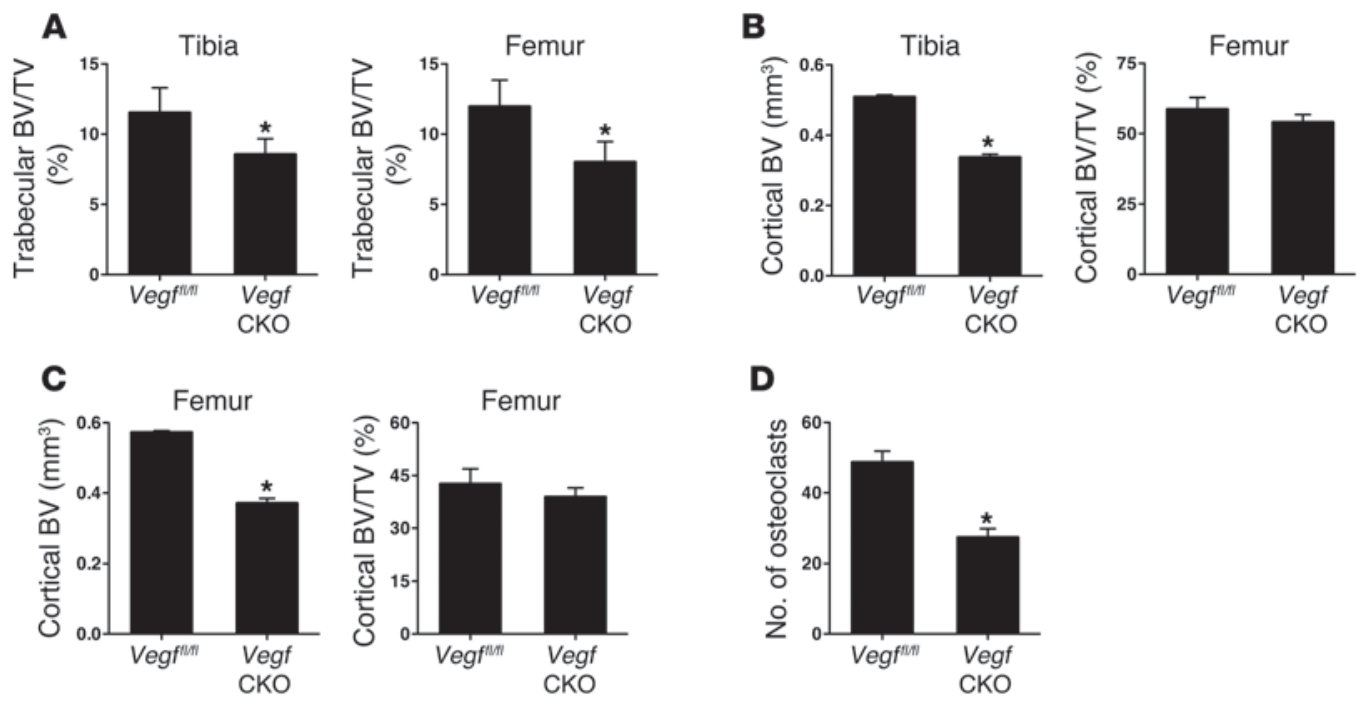

D

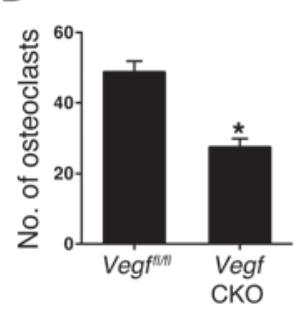

E
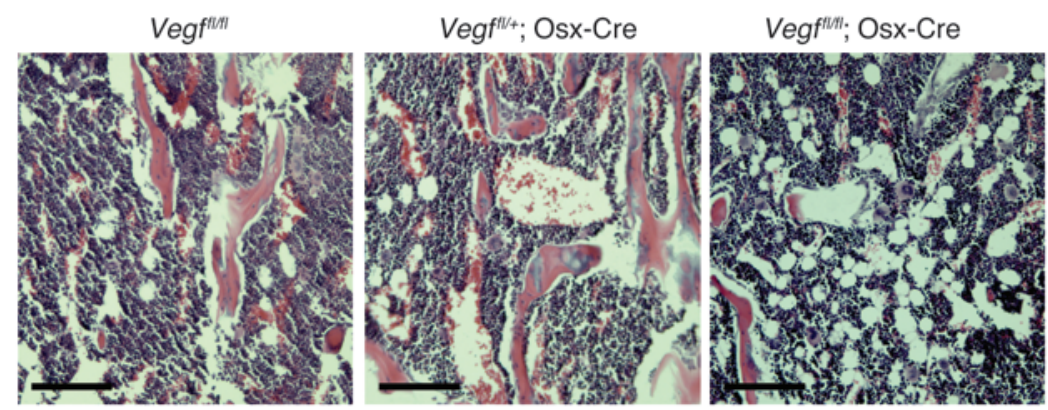

\section{Figure 2}

Reduced trabecular bone and increased bone marrow fat in Vegfa CKO mice. (A) Reduced trabecular bone volume (BV) as percentage of total volume (TV) in tibia and femur of Vegfa CKO mice. ${ }^{*} P<0.05 ; n=3$. (B) MicroCT analysis showed significantly $\left({ }^{\star} P<0.05\right)$ reduced cortical bone volume, but no difference in cortical bone volume as percentage of total volume, in tibia of Vegfa CKO and control mice; $n=3$. (C) MicroCT analysis showed significantly $\left({ }^{\star} P<0.05\right)$ reduced cortical bone volume, but no difference in cortical bone volume as percentage of total volume, in femur of Vegfa CKO versus control mice; $n=3$. (D) Reduced number of TRAP-positive osteoclasts in regions of tibia immediately adjacent to the growth plates (up to $160 \mathrm{~mm}$ from the growth plate) of 2 -month-old mutant mice. ${ }^{*} P<0.05 ; n=3$. (E) Reduced trabecular bone and increased number of adipocytes in sections (H\&E) from metaphyseal regions of tibia of Vegfa CKO (right) compared with control Vegfa ${ }^{f / f l}$ (left) and Vegfa ${ }^{f l+} ;$ Osx-Cre (middle) mice. No difference was observed in trabecular bone and bone marrow fat content in sections from Vegfa ${ }^{\text {fllfl }}$ mice and heterozygous Vegfa ${ }^{f l+} ;$ Osx-Cre mice. Scale bars: $150 \mu \mathrm{m}$.

Vegfafl/+;Osx-Cre mice (Figure 2E). Furthermore, histomorphometric analyses of the proximal tibial region showed no difference in several dynamic and static parameters between Vegfafl/fl and OsxCre mice (Supplemental Table 1; supplemental material available online with this article; doi:10.1172/JCI61209DS1). We therefore used Vegfaflfl mice as control mice in the following studies. We analyzed both males and females by histology and found no sex differences regarding reduced trabecular bone density and increased bone marrow fat. To reduce variation, we used only male littermates for all the experiments reported here. Histomorphometry of the proximal tibial region in 8-week-old Vegfafl/fl and Vegfal/fl; OsxCre mice confirmed that deletion of VEGF in the osteoblast lineage resulted in a significant decrease in trabecular bone volume (Figure 3A) and trabecular number (Figure 3B) in Vegfallffl; Osx-Cre mice. Bone formation rate when expressed per bone surface (Figure 3D) was not affected, but bone formation rate per tissue volume was decreased (Figure 3E). Similar to bone formation rate, there was no change in osteoblast surface per bone surface and osteoblast number per bone perimeter (Supplemental Table 2). How- ever, osteoblast number per tissue area was significantly reduced (Figure 3C), reflecting an overall decrease in osteoblast numbers relative to total bone marrow. In contrast, deletion of Vegfa resulted in 13-fold increased adipocyte numbers (Figure 3F) in the bone marrow of Vegfal/fl; Osx-Cre mice. TRAP staining showed no change in osteoclast surface and osteoclast number within the proximal tibia secondary spongiosa (Supplemental Table 2) used for histomorphometry, although a clear trend toward decreased numbers in the mutant was apparent. These data are consistent with the possibility that the decreased trabecular bone volume observed in Vegfa CKO mice is mainly the consequence of a decrease in overall osteoblast number. In addition, the data suggest that deletion of VEGF in the osteoblast lineage stimulates differentiation of mesenchymal stem cells to adipocytes.

Reduced VEGF expression in cells of the osteoblast lineage leads to reduction in osteoblastogenesis and increased adipocyte differentiation in bone marrow cell cultures. To address the possibility that reduced osteoblastic VEGF expression may result in reduced osteoblast formation by mesenchymal stem cells in the trabecular bone marrow, 
A
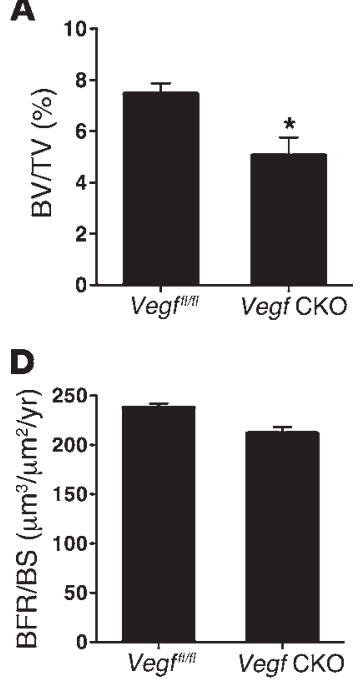

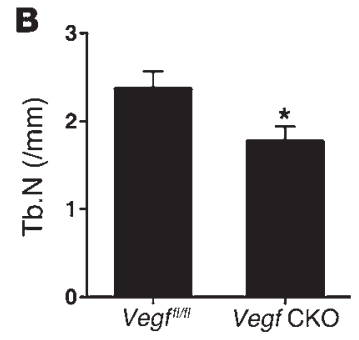

E

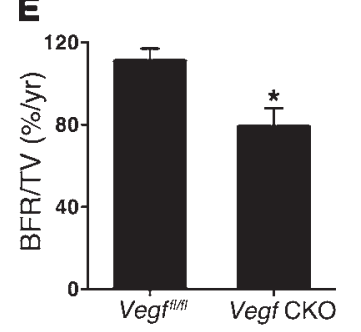

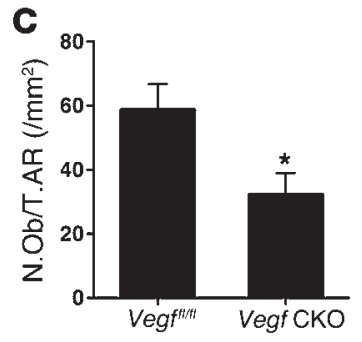

F

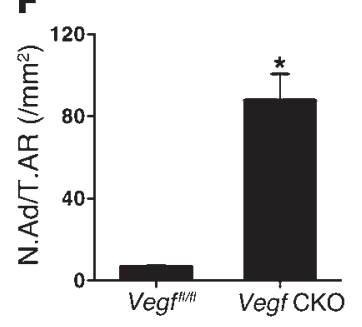

Figure 3

Histomorphometric analysis of control and Vegfa CKO mice. (A) Reduced bone volume/tissue volume in Vegfa CKO mice. ${ }^{*} P<0.05$ versus control mice. (B) Reduced trabecular number (Tb.N) in Vegfa CKO mice. ${ }^{*} P<0.05$ versus control mice. (C) Reduced osteoblast number per tissue area (N.Ob/T.Ar) in Vegfa CKO mice. ${ }^{*} P<0.05$ versus control mice. (D) No difference in bone formation rate was observed when expressed per bone surface (BFR/BS) between control and Vegfa CKO mice. (E) Reduced bone formation rate per tissue volume (BFR/TV) in Vegfa CKO mice. ${ }^{*} P<0.05$ versus control mice. (F) Dramatically increased adipocyte number per tissue area in Vegfa CKO mice. ${ }^{*} P<0.01$ versus control mice. See also Supplemental Table 2. we performed colony-forming unit-fibroblast/osteoblast (CFU-F) assays with bone marrow cells from femur and tibia of mutant and control mice. In these assays, bone marrow cells are cultured at a relatively low density, allowing mesenchymal (stromal) cells to adhere and proliferate to form colonies. Each colony represents the clonal expansion of one mesenchymal cell; thus, the assay reflects the number of mesenchymal cells present in the original bone marrow cell suspension and their clonogenic potential. The number of alkaline phosphatase-positive (ALP-positive) colonies is proportional to the number of progenitors with osteoblastic potential within the mesenchymal cell population (36).

To ensure that the colonies in the CFU-F assays with bone marrow cells obtained from VEGF mutant mice were targeted by OsxCre, we first performed the assays with bone marrow cells from Vegfaflffl; Osx-Cre;Rosa26LacZ mice. Staining of the colonies for LacZ (data not shown) revealed that the colonies were positive for LacZ, indicative of Cre activity. As shown in Figure 4, A-F, the number of ALP-positive colonies was reduced in CFU-F cultures of bone marrow cells derived from Vegfa CKO mice. When cultures were seeded with total bone marrow cells, a dramatic reduction in both the total number of colonies and the fraction of colonies that were positive for ALP was seen in mutant cultures compared with controls. The total number of colonies stained with methylene blue in cultures from mutant mice was reduced to $46 \%$ of that with control cultures from littermates at day 12 (Figure 4C). Only 14\% of the colonies were ALP positive in mutant cultures, compared with $27 \%$ in control littermate cultures (Figure 4B). By day 18, the total number of colonies from mutant mice stained with methylene blue was reduced to $58 \%$ of that with control littermate cultures (Figure $4 \mathrm{~F}) ; 25 \%$ of the colonies were ALP positive in mutant cultures, while $42 \%$ were positive in control cultures (Figure 4E). Addition of recombinant VEGF to the cultures (at concentrations of $20 \mathrm{ng} / \mathrm{ml}$ or higher, see Methods) had no effect on the total number or the percentage of ALP-positive colonies (Figure 4, A-F). Furthermore, addition of neutralizing antibody against VEGF (at concentrations 5 times higher than the dose required for reducing VEGF164induced migration of human umbilical vein endothelial cells to $50 \%$ did not change the number of ALP-positive colonies (Figure 4, $\mathrm{G}$ and $\mathrm{H}$ ). To ensure that concentrations of recombinant VEGF and neutralizing antibodies against VEGF were optimal for detect- ing effects of extracellular VEGF in the bone marrow cell cultures, we also assayed for osteoclast differentiation. VEGF is known to stimulate osteoclast formation in a paracrine manner $(37,38)$, and as expected, the number of TRAP-positive osteoclasts was significantly reduced in cultures of total mutant bone marrow cells compared with control cells (Figure 4, I and J). Addition of recombinant $\operatorname{VEGF}(20 \mathrm{ng} / \mathrm{ml})$ to mutant cultures increased osteoclast numbers to control values (Figure 4, I and J); addition of neutralizing antibodies against VEGF to control cultures reduced osteoclast formation to the levels of mutant cell cultures (Figure 4K).

To address the possibility that the reduced number of CFU-F colonies in bone marrow cultures from mutant mice could be the result of a systemic effect in vivo prior to harvesting of the bone marrow, we isolated total bone marrow cells from control Vegfafl/fl mice and knocked down VEGF expression in vitro by infection with Cre adenovirus. In CFU-F assays using these cells, results were similar to those obtained when bone marrow cells were isolated from Vegfa CKO mice (Figure 5A). Addition of recombinant VEGF to cultures treated with Cre adenovirus and of neutralizing antibody against VEGF to cultures treated with GFP adenovirus did not change the number of ALP-positive colonies (Figure 5A).

To exclude the possibility that the effects of VEGF deficiency in the CFU-F assays with total bone marrow cells may be mediated by hematopoietic cells in the bone marrow cultures, we isolated mesenchymal (stromal) cells (BMSCs) from bone marrow of control WT mice, treated the cells with lentiviral control shRNA or VEGF-specific shRNA, and performed CFU-F assays following selective growth in puromycin-containing medium. In these highly enriched BMSC cultures, $70 \%$ of the colonies were ALP positive when the cells were treated with control shRNA, and this was reduced to $33 \%$ when the cells were treated with VEGF shRNA (Figure 5, B and C). This reduction (about 50\%) in the number of ALP-positive colonies was similar to the reduction in the number of ALP-positive colonies when cultures of total bone marrow cells from Vegfafl/fl and Vegfafl/fl; Osx-Cre mice were compared (Figure 4, $\mathrm{B}, \mathrm{C}, \mathrm{E}$, and F), providing strong evidence that the CFU-F assays described above represent a robust measure of the effect of VEGF expression on the osteoblastic differentiation potential of BMSCs.

Since histology and morphometry demonstrated not only decreased trabecular bone volume but also a substantial increase 
A

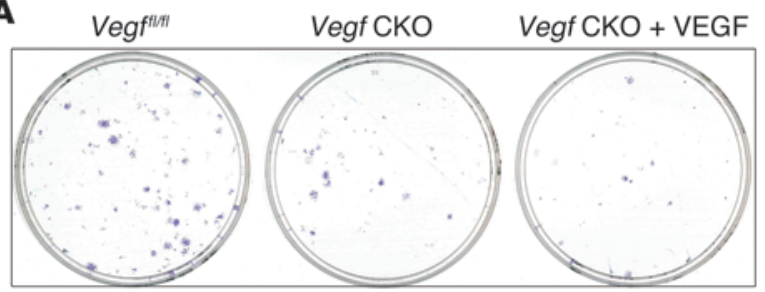

D

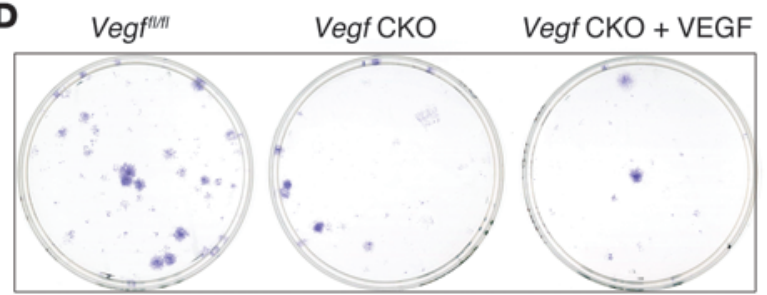

B

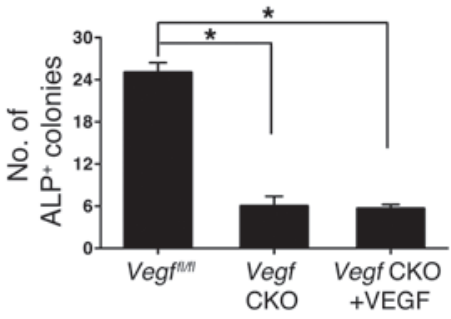

E

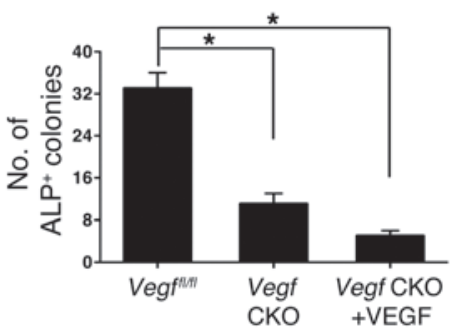

C

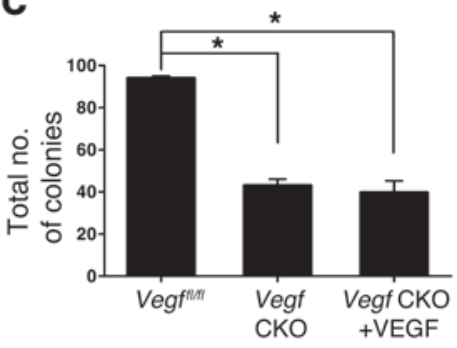

$\mathbf{F}$

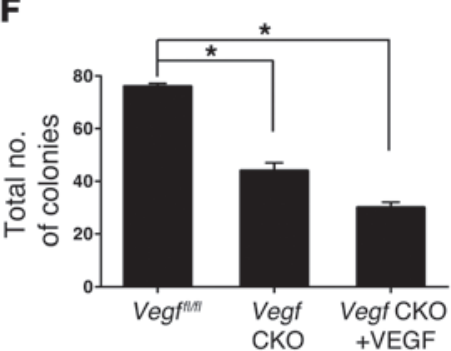

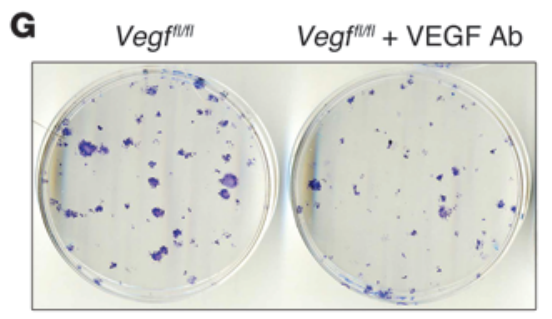

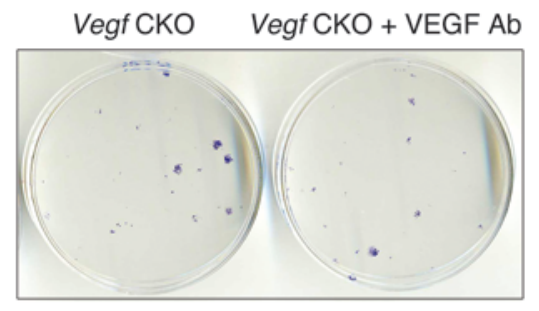

$\mathbf{H}$
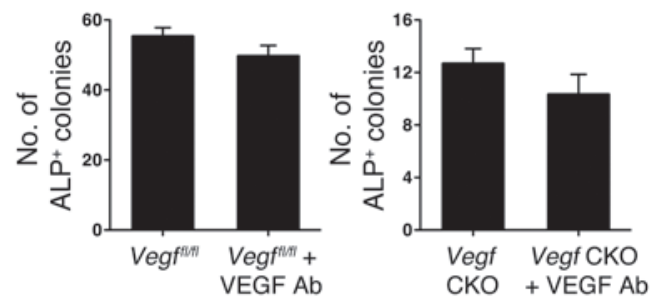

I

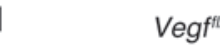

Vegf CKO

Vegf CKO + VEGF
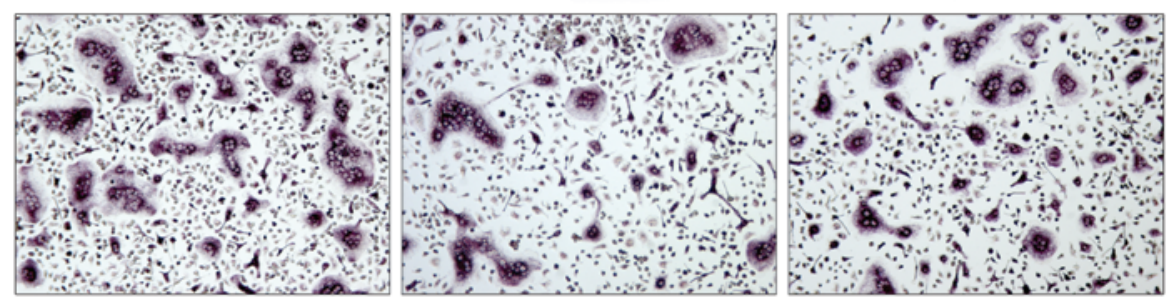

J

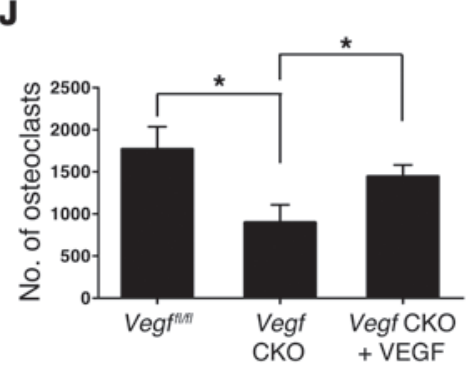

K
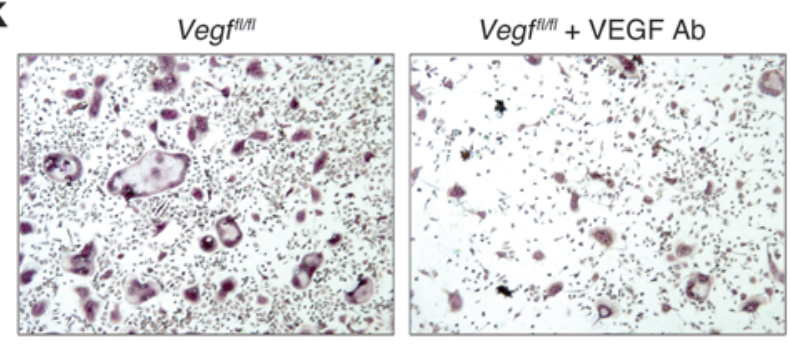

Figure 4

Suppressed osteoblastogenesis resulting from VEGF deficiency in CFU-F assays. Reduced number of ALP-positive colonies in CFU-F assays from Vegfa CKO (middle) compared with control (left) mice at day 12 (A) and day 18 (D). Staining for ALP activity using fast blue BB. Numbers of colonies with cells from mutant mice are the same in the absence and presence of exogenous VEGF (20 ng/ml) (right) in the CFU-F assay. (B and E) Comparison of colony number in the CFU-F assays shown in $\mathbf{A}$ and $\mathbf{D}$. ${ }^{*} P<0.01 ; n=3$. (C and $\mathbf{F}$ ) Total colonies (stained with methylene blue) in CFU-F assays at day 12 and day $18 .{ }^{*} P<0.001 ; n=3$. (G) VEGF neutralizing antibody $(0.8 \mu \mathrm{g} / \mathrm{ml})$ does not reduce the number of ALP-positive (fast blue BB-stained) colonies. (H) VEGF neutralizing antibody does not reduce the number of ALP-positive colonies in the assays shown in G; $n=3$. (I) Osteoclastogenesis with bone marrow cells from Vegfa CKO mice (middle) is reduced compared with cells from control mice (left). Adding VEGF $(20 \mathrm{ng} / \mathrm{ml})$ to the mutant culture restores osteoclastogenesis to control levels (right). Original magnification, $\times 10$. (J) Quantitation of osteoclastogenesis data as presented in I. (K) Osteoclastogenesis with bone marrow cells from control mice is reduced when neutralizing VEGF antibodies $(0.8 \mu \mathrm{g} / \mathrm{ml})$ are added to the culture. Original magnification, $\times 10$. 
A

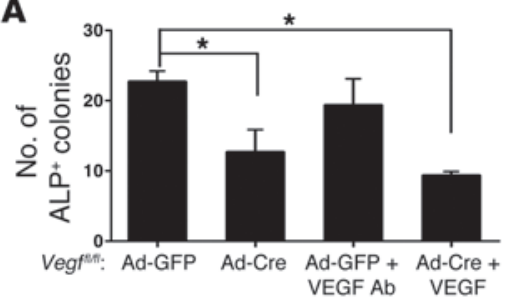

B

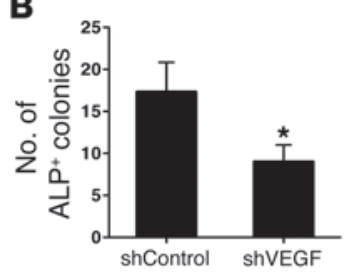

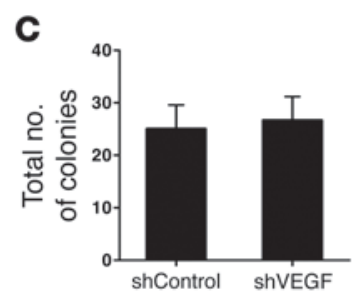

$\mathbf{E}$
D
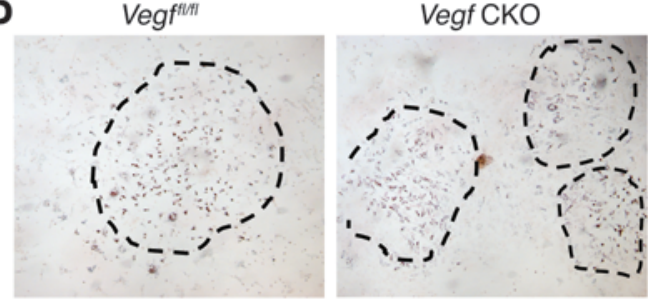

$\mathbf{F}$

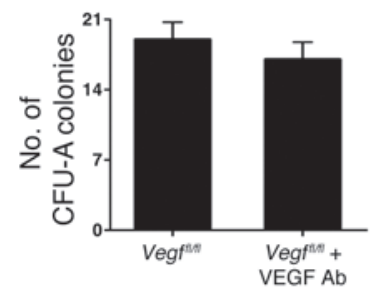

G

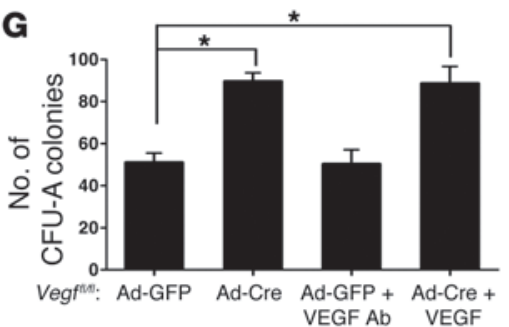

H

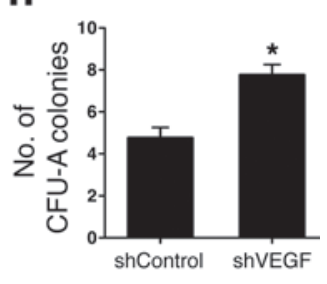

Figure 5

Loss of VEGF in osteoblast lineage increases adipogenesis. (A) Reduced numbers of ALP-positive colonies following addition of Ad-Cre to bone marrow cells from Vegfa fl/fl mice compared with control Ad-GFP. The number of colonies is not affected by exogenous VEGF $(20 \mathrm{ng} / \mathrm{ml})$ or neutralizing antibody $(0.8 \mu \mathrm{g} /$ $\mathrm{ml}$ ). ${ }^{*} P<0.01 ; n=3$. (B) Reduced number of ALP-positive colonies in bone marrow cultures expressing VEGF shRNA compared with control shRNA. ${ }^{\star} P<0.05 ; n=3$. (C) The total number of colonies is not affected by treatment of cultures with VEGF-specific shRNA; $n=3$. (D) Adipocyte colonies (boundaries indicated by dashed lines) in assays used for colony counts. Original magnification, $\times 10$. (E) More adipocyte colonies in CFU-A assays with BMSCs of mutant mice (middle) in adipogenic medium than with cells from control littermates (left). Addition of $20 \mathrm{ng} / \mathrm{ml}$ VEGF has no significant effect. ${ }^{*} P<0.01$ compared with Vegfa fl/fl control; $n=3$. (F) No effect of neutralizing antibody $(0.8 \mu \mathrm{g} / \mathrm{ml})$ against VEGF in CFU-A assays with cells from control mice. (G) Increased number of adipocyte colonies in assays with bone marrow cells from Vegfafl/fl mice treated with Ad-Cre compared with Ad-GFP. No effect of exogenous VEGF $(20 \mathrm{ng} / \mathrm{ml})$ or neutralizing antibody $(0.8 \mu \mathrm{g} / \mathrm{ml}) .{ }^{*} P<0.01 ; n=3$. $(\mathbf{H})$ Increased number of adipogenic colonies in cultures expressing VEGF shRNA compared with control shRNA. ${ }^{*} P<0.001 ; n=4$.

lentivirus encoding control shRNA or VEGF-specific shRNA, selected the cells in puromycin-containing medium, and performed CFU-A assays in adipogenic medium. In these highly enriched BMSC cultures, the number of adipocyte colonies was significantly increased when the cells expressed VEGF-specific shRNA (Figure 5H).

Mechanisms by which VEGF stimulates osteoblast and represses adipocyte differentiation. Our finding that exogenous VEGF or neutralizing antibody against VEGF affects neither the number of ALP-positive CFU-F colonies nor the number of adipocyte colonies in CFU-A cultures raises the possibility that VEGF controls the balance between osteoblastogenesis and adipogenesis through an intracrine mechanism. If this is correct, retrovirusmediated expression of a Vegfa gene in mutant BMSCs should rescue reduced osteoblastogenesis in CFU-F and increased adipogenesis in CFU-A assays. When we infected BMSCs - isolated from Vegfallfl mice and treated with Cre adenovirus - with VEGF retrovirus (Re-VEGF) or control GFP retrovirus (Re-GFP), the retrovirusmediated expression of VEGF164 rescued the defects in CFU-F and CFU-A cultures due to Cre adenovirus-induced loss of VEGF expression (Figure 6, A and B).

These data suggest that osteoblast-generated VEGF regulates the number of CFU-F and CFU-A colonies in bone marrow cell cultures via intracrine mechanisms that do not involve extracellular VEGF. To in the number of adipocytes in bone marrow of Vegfa CKO mice, we next addressed the cellular mechanism of increased adipocyte formation. In vitro colony forming unit-adipocyte (CFU-A) assays showed that mutant BMSCs in adipogenic medium formed more adipocyte colonies than cells from control littermates (Figure 5, $\mathrm{D}$ and $\mathrm{E}$ ). Addition of exogenous VEGF or neutralizing antibody against VEGF had no effect on the number of adipocyte colonies in these cultures (Figure 5, E and F), indicating that VEGF-dependent mechanisms underlying differentiation of adipocytes from BMSCs are not affected by extracellular VEGF. To exclude the possibility that increased adipogenesis in mutant mice is caused by a systemic effect in vivo prior to harvesting the bone marrow, we isolated BMSCs from control mice and knocked down VEGF levels in vitro using Cre adenovirus. When we analyzed adipocyte formation using these cells, results were similar to those obtained with BMSC cultures from mutant mice (Figure 5G). Finally, we treated BMSCs with address the question of whether these mechanisms include interaction of VEGF with its receptors, we first used the Osx-Cre strategy to conditionally target Flt1 and Flk1 in osteoblastic lineage cells. Histological examination of mutant skeletons revealed decreased trabecular bone in both mutants, but no increase in bone marrow adipocyte numbers (data not shown). As expected, based on the in vivo bone phenotypes, examination of ALP-positive CFU-F colonies in total bone marrow cell cultures from both Flt1 and Flk1 CKO mice showed that the number of positive colonies was reduced when compared with cultures from control littermates (Figure 6, C and D). In contrast, knocking down Flk1 expression had no effect on the number of adipocyte colonies in CFU-A assays and knocking down Flt1 reduced the number of adipocyte colonies compared with controls (Figure 6, E and F). Thus, both receptors are required for the stimulatory effect of VEGF on osteoblastogenesis, but they are not critical for the ability of VEGF to repress adipocyte differentiation. 

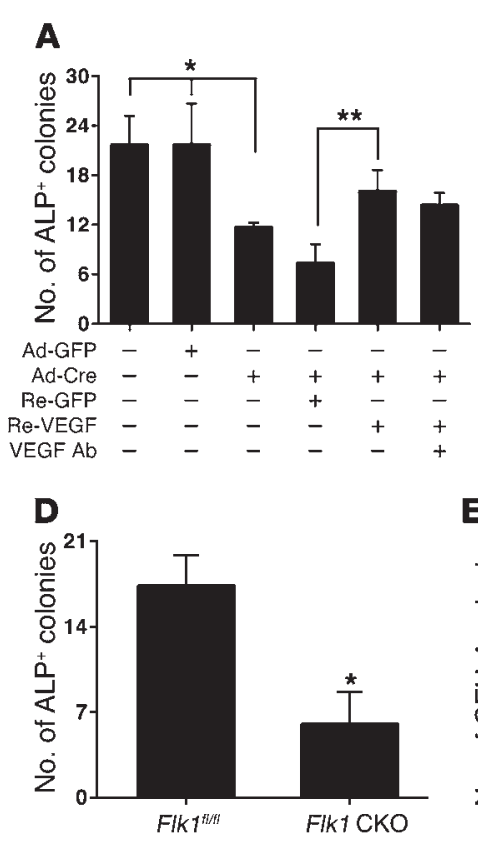

B

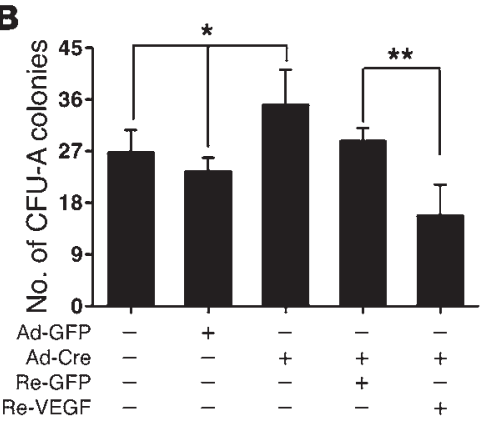

C

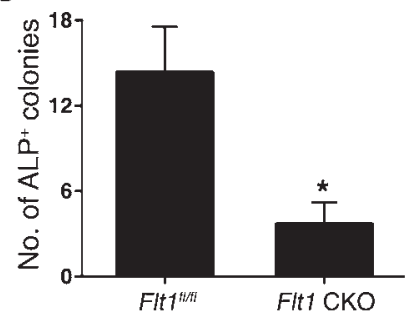

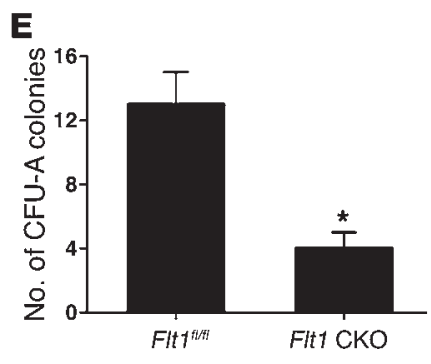

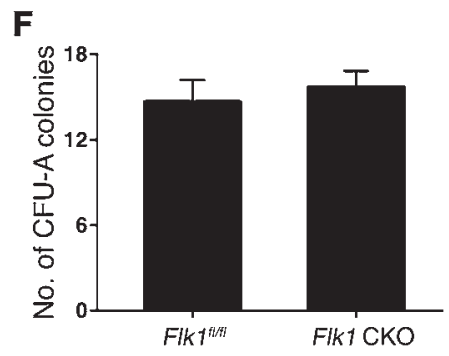

\section{Figure 6}

Virus-mediated expression of VEGF rescues osteoblast and adipocyte differentiation in VEGF-deficient cells. (A) Infection with VEGF retrovirus (Re-VEGF) rescues the reduction in colony number in CFU-F assays of Vegfa ${ }^{f l / f l}$ cells treated with Ad-Cre compared with control retrovirus (ReGFP). No effect of neutralizing antibody against VEGF $(0.8 \mu \mathrm{g} / \mathrm{ml}) .{ }^{*} P<0.01,{ }^{*} P<0.05 ; n=3$. (B) Decreased number of colonies in CFU-A assays when BMSCs, isolated from Vegfa ${ }^{f / f l}$ mice and treated with Cre adenovirus, are infected with VEGF retrovirus as compared with control retrovirus. ${ }^{*} P<0.05,{ }^{* *} P<0.01 ; n=3$. (C and $\left.\mathbf{D}\right)$ Reduced number of ALP-positive colonies in CFU-F assays of bone marrow cells from conditional VEGF receptor-knockout mice. (C) Reduced number of colonies with cells from Flt1 mutants. ${ }^{*} P<0.01 ; n=3$. (D) Reduced number of colonies with cells from Flk1 mutants. ${ }^{*} P<0.01 ; n=3$. (E and F) Number of adipocyte colonies in CFU-A assays of bone marrow cells from conditional VEGF receptor-knockout mice. (E) Reduced number of colonies with cells from Flt1 mutants. ${ }^{*} P<0.01 ; n=3$. (F) No changes in colony number with cells from Flk1 mutants.

Next, we stained BMSCs with antibodies against VEGF, VEGFR1, and VEGFR 2 to determine whether the 3 proteins are colocalized within particular intracellular compartments. As shown in Figure 7A, positive staining for VEGF and VEGFR2 was detected in both nuclear and cytoplasmic regions, but staining for VEGFR1 was primarily observed in the nuclear area, as demonstrated by the similarity to the staining pattern seen when BMSCs were immunostained for the nuclear envelope protein lamin A/C. Based on this observation and our finding that osteoblasts isolated from femur and tibia of Vegfallfll; Osx-Cre mice exhibit the flattened phenotype of senescent cells and stain positively for senescence-associated $\beta$-galactosidase (data not shown), we considered a potential association between lamin A/C and intracrine VEGF signaling. Depletion of lamin A/C induces cellular senescence (39), and knockdown of lamin A/C in BMSCs has been reported to reduce osteoblastic differentiation, increase adipocyte differentiation, and lower RUNX2 DNA binding activity (40-44). Furthermore, lamin A-null mice exhibit a postnatal decline in bone mass (44). Given the similarity of these characteristics to those of mice lacking VEGF in osteoblastic lineage cells, we considered the possibility that the effect of VEGF on osteoblastogenesis may be mediated by a mechanism that includes a functional interaction between VEGF and lamin A/C.

First, we used quantitative assays for VEGF and Western blotting for lamin A/C to analyze cellular lysates obtained from BMSCs of control (WT) and heterozygous lamin A/C-null $\left(\mathrm{Lmna}^{+/-}\right)$mice treated with lentiviral control shRNA or VEGF- specific shRNA. As shown in Figure $7 \mathrm{~B}$, the concentration of cell-associated VEGF was reduced to almost the same extent in lysates obtained from cells expressing VEGF-specific shRNA and control shRNA-expressing cells from $L m n a^{+/-}$mice. In addition, Western blotting demonstrated that protein levels of lamin A (Figure 7C) were increased in cells expressing VEGF-specific shRNA. These data suggest that while lamin A stimulates VEGF protein expression, VEGF represses levels of lamin A. When the lentivirus-treated cells were used for CFU-F assays, we found that $\mathrm{Lmna}^{+/-}$cells generated more total colonies than WT cells (Figure $7 \mathrm{E})$, consistent with published evidence that lamin A/C represses proliferation of mesenchymal cells (45-47). However, the fraction of ALP-positive colonies in the assays was proportional to the level of VEGF protein in the cells (Figure 7, B, D, and E). These data indicate that cell-associated VEGF is a major stimulator of differentiation of BMSCs along the osteoblastic pathway. In contrast, cell-associated VEGF represses differentiation of BMSCs to adipocytes. When CFU-A assays were carried out using lentivirustreated BMSCs from WT and $L m n a^{+/-}$mice, the relative number of adipocyte colonies increased dramatically when the levels of VEGF in cell lysates at the start of the CFU-A assay were low (Figure 7F). However, the VEGF levels in the culture medium were hardly affected when cells expressed VEGF-specific shRNA and showed no correlation with adipocyte colony numbers (Figure $7 \mathrm{G})$. The number of adipocyte colonies as a fraction of total colonies (about 30\%) was similarly increased in WT cultures express- 
A
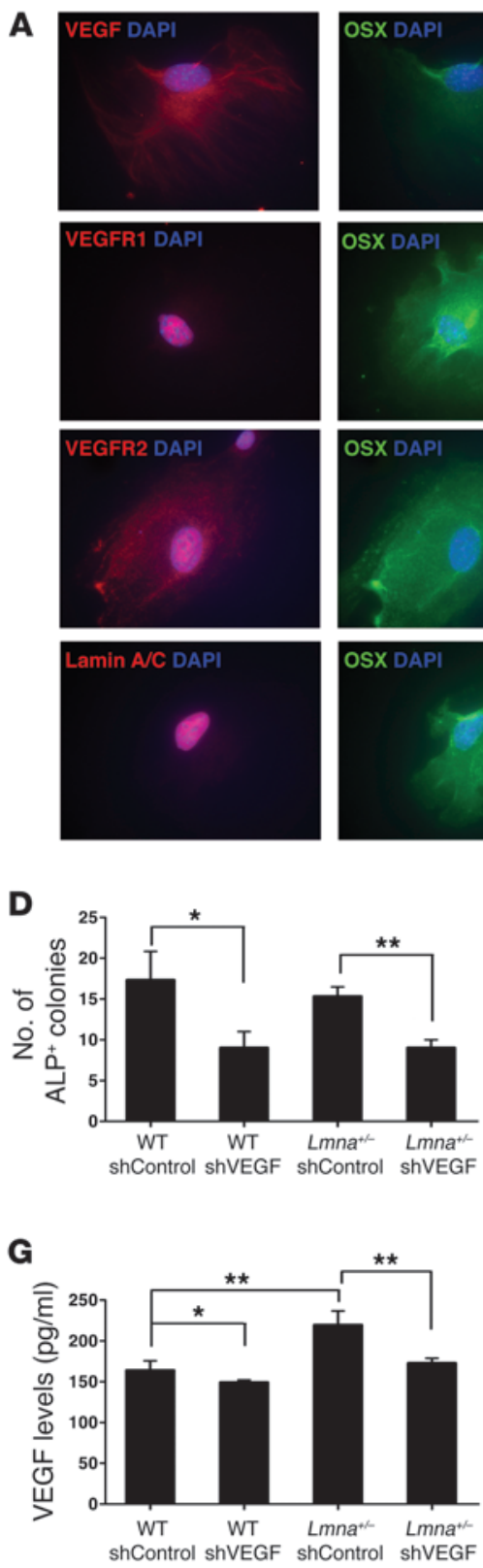

K

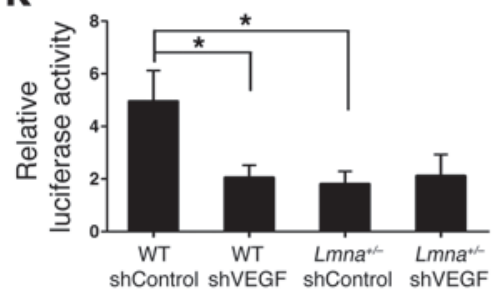

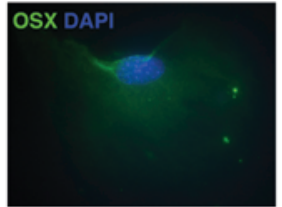
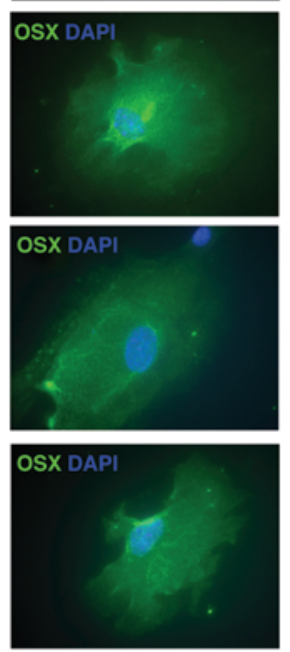
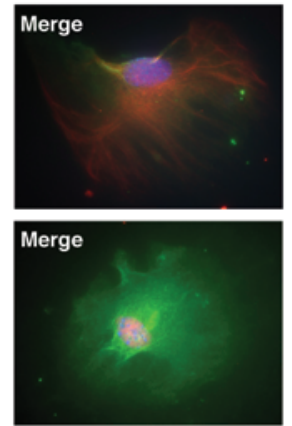

\section{Merge}

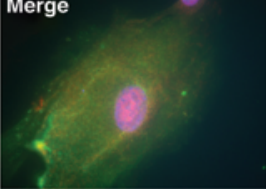

Merge

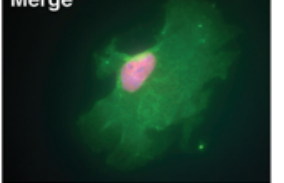

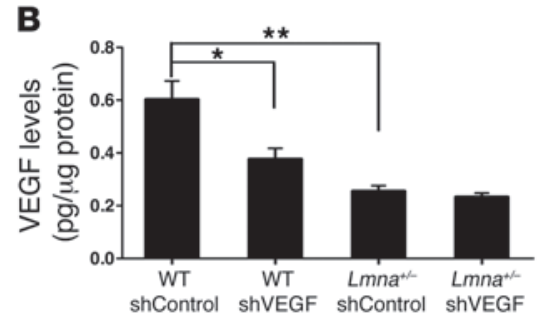

C

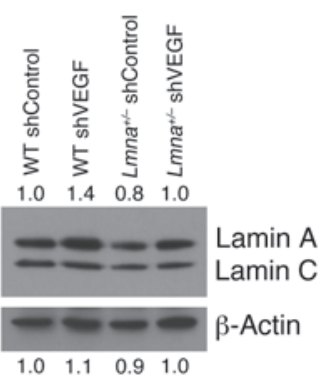

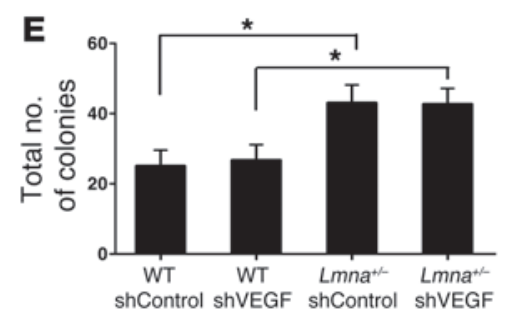

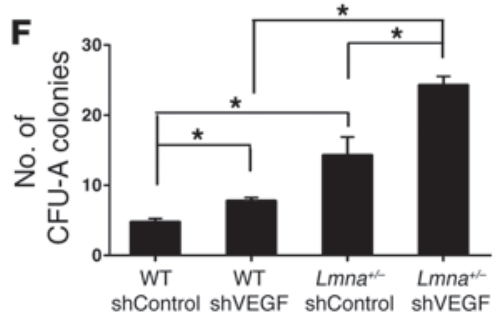

H

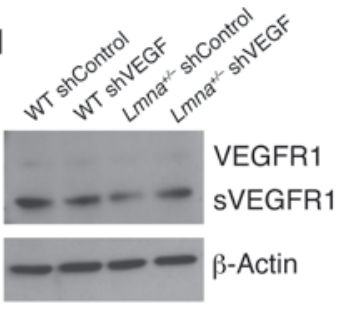

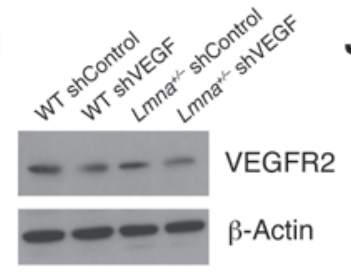

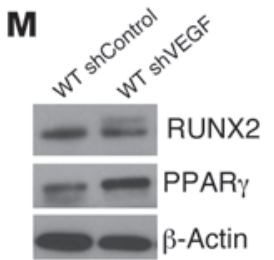

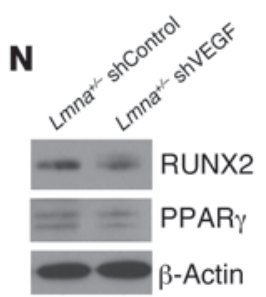

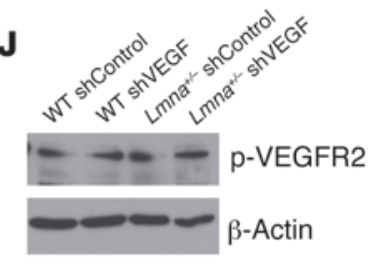

$\mathbf{L}$

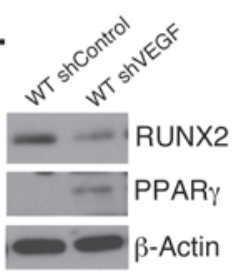

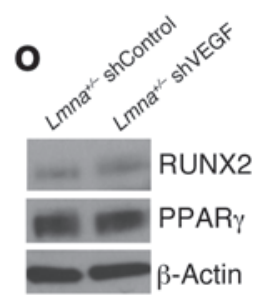




\section{Figure 7}

Mechanisms by which VEGF controls osteoblast/adipocyte differentiation in BMSCs. (A) VEGF and VEGFR2 expression is detected in both nuclear and cytoplasmic regions of Osx-expressing cells, whereas VEGFR1 expression is primarily observed in the nuclear area similar to lamin A/C. Original magnification, $\times 250$. (B) Reduced VEGF protein levels associated with WT and $L m n a^{+/-}$cultures expressing VEGFspecific shRNA. ${ }^{*} P<0.05,{ }^{* *} P<0.01 ; n=3$. (C) Increased lamin $A$ protein levels associated with WT and $L m n a^{+/}$cultures expressing VEGF-specific shRNA. Values above and below the blots represent relative lamin $A$ and $\beta$-actin protein levels. (D) Reduced number of ALP-positive colonies in both WT and $L m n a^{+/-}$cultures expressing VEGF shRNA. ${ }^{\star} P<0.05,{ }^{* \star} P<0.01 ; n=3$. (E) The number of colonies increased in $L m n a^{+/}$compared with WT cultures but was not affected by treatment with VEGF-specific shRNA. ${ }^{*} P<0.05 ; n=3$. (F) Increased number of adipogenic colonies in WT and $L \mathrm{mna}^{+/-}$ cultures expressing VEGF shRNA and $\mathrm{Lmna}^{+/-}$cultures expressing control shRNA. ${ }^{*} P<0.001 ; n=4$. (G) VEGF protein levels in culture medium prior to CFU-A assay are only slightly affected in WT and Lmna ${ }^{+/-}$cultures expressing VEGF-specific shRNA. ${ }^{*} P<0.05$ and ${ }^{* *} P<0.01$. (H) VEGFR1 is mainly expressed as the soluble splice variant (sVEGFR1), whereas full-length (I) and phosphorylated (J) VEGFR2 is detected in WT and $\mathrm{Lmna}^{+/-}$cultures. (K) Reduced RUNX2 transcriptional activity in cultures expressing VEGF-specific shRNA and/or $L m n a^{+/-}$cultures expressing control shRNA. ${ }^{*} P<0.05$; $n=4$. ( $\mathbf{L}$ and $\mathbf{N})$ RUNX2 protein levels are decreased in WT (L) and $\mathrm{Lmna}^{+/-}(\mathbf{N})$ cultures expressing VEGF-specific shRNA under osteogenic conditions. ( $\mathbf{M}$ and $\mathbf{0}$ ) PPAR 2 protein levels are increased in WT $(\mathbf{M})$ and $L m n a^{+/-}(\mathbf{O})$ cultures expressing VEGF-specific shRNA in the presence of adipogenic inducers.

ing VEGF-specific shRNA and in cultures of $\mathrm{Lmna}^{+/-}$cells expressing control shRNA. The level of cell-associated, but not secreted, VEGF was significantly reduced in these two cultures, whereas the level of lamin A was increased in cultures expressing VEGF shRNA. These data suggest that lamin A does not affect adipocyte differentiation in a VEGF-independent manner.

Since both VEGFR1 and VEGFR2 are required for osteoblastic differentiation in mesenchymal stem cells, we analyzed the protein levels of the two receptors in lysates of lentivirus-treated BMSCs. As shown in Figure 7, I and J, full-length and phosphorylated VEGFR2 protein was found in all lysates, while the major form of VEGFR1 protein was the soluble splice variant (sVEGFR1), with only trace amounts of the full-length form detected (Figure $7 \mathrm{H}$ ). Given the high affinity of sVEGFR1 for VEGF and the nuclear localization of VEGFR1 in BMSCs, this raises the intriguing possibility that sVEGFR1 may serve to concentrate VEGF in the perinuclear, lamin A/C-containing region in the cells. Finally, given findings that reduced levels of lamin $\mathrm{A} / \mathrm{C}$ result in reduced RUNX2 activity $(41,44)$, we used a luciferase reporter assay to determine RUNX2 transcriptional activity in lentivirus-treated BMSCs cultured in osteogenic medium. The results of these assays (Figure $7 \mathrm{~K}$ ) indicated that the activity is significantly reduced when cells were expressing VEGF-specific shRNA and/or were heterozygous for lamin A-null alleles. Furthermore, when lentivirus-treated BMSCs were cultured in osteogenic and adipogenic medium, the protein level of RUNX2 was decreased while the level of the lipidactivated transcription factor PPAR $\gamma 2$ (48) was increased in lysates of cells expressing VEGF-specific shRNA compared with control shRNA (Figure 7, L-O). These data indicate that intracrine VEGF in BMSCs stimulates osteoblastic differentiation in a RUNX2dependent manner and represses levels of PPAR $\gamma 2$.

\section{Discussion}

In this study, VEGF was conditionally targeted in Osx-positive osteoblast lineage cells, allowing studies of the functions of osteoblast-derived VEGF. Our data demonstrate that osteoblastderived VEGF stimulates formation of osteoblasts and suppresses adipogenesis, both in vivo and in vitro. Thus, normal postnatal bone homeostasis requires expression of VEGF in osteoblasts. The reduction in bone mass and the increase in marrow fat in Vegfa CKO mice is similar to changes associated with osteoporosis and age-related osteopenia (4). Postmenopausal women with polymorphisms associated with high or low VEGF production have higher or lower lumbar spine bone mineral density, respectively (49). Intriguingly, there also appears to be no significant association between levels of circulating VEGF and bone mineral density (49). This is consistent with our in vitro data demonstrating that addition of recombinant VEGF to cultures of VEGF-deficient BMSCs, even at very high concentrations, has no effects on the differentiation properties of the mutant cells. This suggests that VEGF controls osteoblastogenesis and adipogenesis via mechanisms that are resistant to the effects of extracellular VEGF.

The concept of an intracrine signaling loop for VEGF has been previously suggested in the case of bone marrow hematopoietic stem cells (50). VEGF appears to control survival of these stem cells via a mechanism that is resistant to inhibitors that fail to penetrate the plasma membrane, such as antibodies (50). A similar phenomenon has been noticed in studies in which VEGF was specifically deleted in endothelial cells (51). Furthermore, a recent report suggests that VEGF increases survival and chemoresistance in human colorectal cancer cells by an intracrine mechanism (52). How intracrine functions of VEGF may be regulated and in which compartments VEGF may function intracellularly has not been known. However, several observations provide intriguing hints. VEGF has been reported to mediate survival of human breast cancer carcinoma cells via binding intracellularly to VEGFR1 (53), and it has been found to accumulate in endothelial cell nuclei (54). The immunocytochemical detection of sVEGFR1, VEGFR2, and VEGF in the nuclear region of BMSCs in the present study is consistent with these observations. A key question is whether the intracrine function in Osx-positive osteoblastic lineage cells requires direct binding of VEGF to its receptors. Our data do not allow a definitive answer to this question. However, since knockdown of either Flt1 or Flk1 in the osteoblast lineage led to reductions in the numbers of ALP-positive CFU-F colonies, interactions of intracellular VEGF with sVEGFR1 and VEGFR2 in differentiating osteoblasts may be part of the mechanism by which VEGF stimulates osteoblastic differentiation in BMSCs. In lysates of BMSCs, VEGFR2 was found to be phosphorylated on Y1175, and downstream targets, such as Akt and p38MAPK, were phosphorylated as well even when cell-associated VEGF protein levels were knocked down (Supplemental Figure 1). Therefore, while the receptors may be involved in the intracrine effects of VEGF on osteoblastic differentiation, it is unlikely that the effects are mediated by signaling downstream of activated VEGFR2. Interestingly, Domingues et al. (55) recently reported that a VEGF/VEGFR2 complex can be transported into nuclei in endothelial cells, where it can interact with several nuclear proteins, including the transcription factor Sp1, and stimulate the expression of VEGFR2. If this mechanism also applies to BMSCs, one would expect the level of VEGFR2 to decrease with decreasing levels of VEGF in the cells, and this is consistent with lower levels of VEGFR2 in lysates from BMSCs treated with VEGF-specific shRNA. 


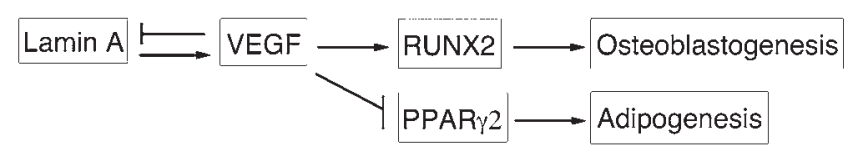

Figure 8

Diagram illustrating a model for the reciprocal functional interactions between VEGF and lamin A and the proposed relationships among the levels of VEGF, RUNX2, and PPARy2 based on the data. VEGF may promote osteoblastogenesis in mesenchymal stem cells by stimulating the level and transcriptional activity of RUNX2 and may control adipogenesis by suppressing the expression of PPAR 2 .

The association between low levels of VEGF and low levels of RUNX2 protein and activity in BMSCs provides an explanation for the reduced ability of the cells to differentiate into osteoblasts when VEGF levels are knocked down. Elucidation of the mechanisms by which VEGF affects RUNX2 expression and activity will require further studies. However, since $\mathrm{Sp} 1$ has been demonstrated to be important for stimulation of Runx2 transcription (56), the findings of Domingues et al. (55) raise the possibility that VEGF/ VEGFR2 complexes may have a role in Sp1-dependent induction of Runx 2 transcription. Combined with evidence that RUNX2 can serve as a transcription factor to induce transcription of Vegfa (35, 57), this suggests the existence of a positive feedback loop between intracrine VEGF and RUNX2 expression.

The suppression of adipocyte differentiation by VEGF does not include VEGFR1- and VEGFR2-dependent signaling mechanisms. Results of CFU-A assays with VEGFR2-deficient BMSCs were not different from those of assays with control cells. Also, VEGFR1 deficiency in BMSCs inhibited adipogenesis. This is consistent with the known function of sVEGFR1 as a high-affinity decoy receptor for VEGF in many contexts; loss of VEGFR1 may increase the efficiency of VEGF as a suppressor of adipogenesis. Thus, other VEGF-binding receptors or receptor-independent VEGF functions may be involved in the mechanism by which VEGF suppresses adipocyte differentiation. Knockdown of lamin A in mesenchymal stem cells results in increased adipocyte differentiation $(42,43)$, raising the question of whether the effect of VEGF on adipocyte differentiation is mediated by lamin A. Our data indicate that this is not the case. Loss of one Lmna allele in BMSCs caused cell-associated VEGF to be reduced to the levels in cells treated with VEGFspecific shRNA, suggesting that lamin A stimulates VEGF protein expression. In addition, the fact that levels of lamin A/C expression increased when WT or Lmna ${ }^{+/-}$mesenchymal cells expressed VEGF-specific shRNA indicates that VEGF, in turn, suppresses lamin A expression. Such a reciprocal relationship between VEGF and lamin A may explain why loss of one Lmna allele in cells results in lamin A levels that are greater than 50\% of what they are in WT cells. The relationship between VEGF and lamin A also indicates that lamin A is upstream of VEGF with respect to both osteoblast and adipocyte differentiation. In fact, our data suggest that most, if not all, effects of lamin A on osteoblast and adipocyte differentiation may be mediated by the effects of lamin A on the level of VEGF in cells (Figure 8).

Activation of Wnt/ $\beta$-catenin signaling suppresses adipogenesis and promotes osteoblastogenesis of mesenchymal stem cells. This raises the question of whether Wnt signaling may be involved in the VEGF-dependent functions in the cells (13-15). However, disruption of canonical Wnt signaling in mice causes bone loss due to reduced osteoblast differentiation, but adipogenesis is not affected (16). Furthermore, transdifferentiation of mesenchymal stem cellderived osteoblast progenitors into adipocytes is not associated with a change in expression of Wnt3a and $\beta$-catenin (58).

In addition to the intracrine mechanism for VEGF function related to osteoblast/adipocyte differentiation as suggested by the data presented here, we also demonstrate that VEGF secreted by BMSCs has paracrine effects on osteoclast differentiation in assays using total bone marrow cell cultures. Furthermore, it is known that osteoblast-derived VEGF can affect bone formation in a paracrine manner via stimulation of angiogenesis (59). It will be interesting to investigate whether and how osteoblast-derived intracrine VEGF affects the interaction between osteoblasts and endothelial cells in bone marrow, since it has been reported that direct contact between endothelial cells and osteoprogenitors in vitro supports osteoblast function (60). To our knowledge, this is the first in vivo/in vitro study to investigate the roles of osteoblastderived VEGF in osteoblastic and adipocytic differentiation. Based on the results of the study, we propose that understanding the mechanisms by which intracrine VEGF controls adipocyte and osteoblast fates may lead to identification of novel therapeutic targets to control bone loss in osteoporosis.

\section{Methods}

Mouse strains. Vegfa floxed and Osx-Cre mouse lines have been described previously $(61,62)$. Flk1 floxed and Flt 1 floxed mice were generated using standard procedures. Genomic DNA isolated from portions of mouse tails were used for VEGF and VEGF receptor genotyping. Genotyping for Vegfa was carried out using PCR with the following primers: forward $5^{\prime}$-CCTGGCCCTCAAGTACACCTT-3'; reverse 5'-TCCGTACGACGCATTTCTAG-3'. For Flt 1 the primers were forward 5'-CCTGCATGATTCCTGATTGGA-3'; reverse 5'-GCCTAAGCTCACCTGCGG-3'. For Flk1 the primers were forward 5'-GACTTGGTTCATCAGGCTAG-3'; reverse 5 '-GACGCTGTTAAGCTGCTACAC- 3 '. The primers for Cre were forward 5'-GATGAGGTTCGCAAGAACCTG-3'; reverse 5'-TGAACGAACCTGGTCGAAATC-3'. The Rosa26LacZ reporter mice and the $L m n a^{+/-}$mice were obtained from the Jackson Laboratory. To assess the efficiency of Osx-Cre-mediated inactivation of Vegfa in bones of mutant mice, RNA was extracted from tibia and femur of newborn control and mutant pups and used for real-time PCR with primers (forward 5 -ATCTTCAAGCCGTCCTGTGT-3' and reverse 5'-CTGCATGGTGATGTTGCTCT-3') from within exon 3 (flanked by loxP sites in the Vegfa gene). Real-time PCR for Gapdh transcripts (forward primer 5'-TGTGTCCGTCGTGGATCTGA-3' and reverse primer $5^{\prime}$-CCTGCTTCACCACCTTCTTGA-3') served as internal control. The results indicated that exon 3-containing Vegfa transcripts in extracts of bones were reduced by about $70 \%$ in mutant bones. Since isolated bones contain non-osteoblastic cells, such as endothelial cells, this value likely underestimates the degree of Osx-Cre-dependent Vegfa deletion in osteoblastic cells.

$X$-ray and microCT. X-ray and microCT analyses were performed using Faxitron Micro 50 X Ray and Scanco Medical $\mu$ CT-40 instruments.

Histology and histochemistry. Mouse limbs were fixed in $4 \%$ paraformaldehyde and embedded in paraffin for standard histology or dehydrated with $20 \%$ sucrose and embedded in Tissue-Tek O.C.T. compound (Sakura) for cryostat sectioning. For lipid staining, frozen sections or cell cultures were fixed with $10 \%$ neutral buffered formalin and stained with filtered oil red $\mathrm{O}$ in $60 \%$ isopropanol. ALP staining of cell cultures was performed using Alkaline Phosphatase kit 86R-1KT (with fast red violet LB) or 86C$1 \mathrm{KT}$ (with fast blue BB) (Sigma-Aldrich) as described in the manufacturer's instructions. TRAP staining of frozen sections for osteoclasts was 
performed using a Leukocyte Acid Phosphatase kit (Sigma-Aldrich). For LacZ staining of colonies, CFU-F colonies were fixed in $70 \%$ ethanol. After staining for ALP, colonies were stained with X-gal solution overnight at $30^{\circ} \mathrm{C}$, washed in water, dried, and photographed. For senescence associated $\beta$-galactosidase (SA- $\beta$-gal) staining, cells were fixed in $0.2 \%$ glutaraldehyde/PBS for 10 minutes at room temperature, washed with PBS, and stained with X-gal solution ( $\mathrm{pH}$ 6.0) at room temperature.

Bone histomorphometry. Eight-week-old Vegfall/fl (5 mice) and Vegfallfl; OsxCre (6 mice) mice were subcutaneously injected with $20 \mathrm{mg} / \mathrm{kg}$ calcein and $40 \mathrm{mg} / \mathrm{kg}$ demeclocycline on days 7 and 2 before necropsy, respectively. Tibias were removed and embedded without demineralization in methyl methacrylate. Undecalcified sections were cut at a thickness of $5 \mu \mathrm{m}$ and mounted unstained for dynamic measurements (i.e., mineral apposition rate [MAR], mineralizing surface (MS/BS) and bone formation rate expressed per bone surface [BFR/BS], bone volume [BFR/BV], and tissue volume referent [BFR/ $\mathrm{TV}])$. Consecutive sections were stained with toluidine blue and TRAP to quantify osteoblast number and osteoclast number, respectively. Adipocyte number was counted using toluidine blue-stained sections. Histomorphometric analysis was performed using the OsteoMeasure system (Osteometrics Inc.), and the results were expressed according to standardized nomenclature (63). A sampling site with an area of approximately $1.2 \mathrm{~mm}^{2}$ was established in the secondary spongiosa of the metaphysis.

Isolation of osteoblastic cells from mouse long bones. Isolation and culture of primary bone cells from tibia and femur of 8- to 12-week-old mice were as described (64). Bone marrow cells were flushed out with PBS, and cleaned diaphyses were cut into small pieces and incubated in bacterial collagenase solution at $37^{\circ} \mathrm{C}$. The bone pieces were cultured in DMEM containing $15 \% \mathrm{FBS}$ and $100 \mu \mathrm{g} / \mathrm{ml}$ ascorbic acid until cells migrating from the bone chips became confluent.

Isolation of bone marrow cells. Bone marrow cells from tibia and femur of 8 - to 12 -week-old mice were flushed out with ice-cold $\alpha$-MEM with nucleosides and cultured in $\alpha$-MEM containing $10 \%$ or $20 \%$ FBS following lysis of red blood cells with RBC Lysis Buffer (eBioscience).

For CFU-F assays, $1 \times 10^{6}$ bone marrow cells were plated on $60-\mathrm{mm}$ dishes in $5 \mathrm{ml} \alpha$-MEM (Invitrogen) containing penicillin/streptomycin, $50 \mu \mathrm{g} / \mathrm{ml}$ ascorbic acid (Sigma-Aldrich), 10-8 $\mathrm{M}$ dexamethasone (Sigma-Aldrich), and $10 \%$ FBS (Atlanta Biologicals). After 12 or 18 days, cultures were fixed with $70 \%$ ethanol and stained for ALP (using fast red violet LB or fast blue BB) or with methylene blue for counting of all colonies. CFU-F colonies were scored by counting clusters containing more than 6 cells in the entire dish. For each experiment, repeated at least 3 times, bone marrow cells from 2-3 mice for each genotype were plated in triplicate dishes.

For CFU-A assays, bone marrow cells were plated at $2 \times 10^{6}$ cells per well in 24-well plates with $\alpha$-MEM containing $20 \%$ FBS. When cells became $70 \%-80 \%$ confluent, adipocyte differentiation medium (DMEM supplemented with $10 \%$ horse serum, $10^{-8} \mathrm{M}$ hydrocortisone, $60 \mu \mathrm{M}$ indomethacin, and $500 \mu \mathrm{M}$ 1-isobutyl-3-methylxantine (IBMX) were added, and cells were cultured for 3-4 days before oil red $\mathrm{O}$ staining and counting adipocyte clusters in each well. Each experiment (more than 3 were performed) included triplicate or quadruplicate wells.

In vitro experiments with BMSCs. Bone marrow cells from tibia and femur of 8- to 12 -week-old mice were cultured in $\alpha$-MEM containing $10 \%$ FBS. Non-adherent cells were removed by replacing the medium after 3 days. The attached BMSCs were used for experiments. Cells from Vegfall/fl mice were treated with Cre adenovirus (Ad-Cre) in vitro to knock down Vegfa expression. GFP adenovirus (Ad-GFP) was used as control.

For experiments requiring retroviral infection, $\mathrm{PWZL}$ Blast VEGF and pWZL Blast GFP plasmids, generated by B. Weinberg, were provided by Addgene (cat. 10909 and 12269). The plasmids were transfected into 293GPG packaging cells using FuGENE 6 (Roche). After 48 hours, superna- tants were collected and used for infection. The BMSCs (isolated from Vegfallfl mice and treated with Ad-GFP or Ad-Cre to knock down Vegfa) were infected with VEGF retrovirus (Re-VEGF) or GFP retrovirus (Re-GFP).

For lentiviral shRNA experiments, bone marrow cells from WT and $L m n a^{+/-}$mice were isolated and treated with VEGF shRNA Lentiviral Particles (sc-36815-V, Santa Cruz Biotechnology Inc.), Control shRNA Lentiviral Particles (sc-108080, Santa Cruz Biotechnology Inc.), or copGFP Control Lentiviral Particles (sc-108084, Santa Cruz Biotechnology Inc.) in the presence of $5 \mu \mathrm{g} / \mathrm{ml}$ Polybrene (sc-134220, Santa Cruz Biotechnology Inc.). To select stable clones expressing the shRNA, cells were treated with $2 \mu \mathrm{g} / \mathrm{ml}$ puromycin dihydrochloride (sc-108071, Santa Cruz Biotechnology Inc.) until resistant colonies were identified and used for subsequent experiments.

ELISA assays. VEGF protein levels in cell lysates were assessed using the Quantikine Mouse VEGF Immunoassay (R\&D Systems) in accordance with the manufacturer's instructions; VEGF levels in lysates from confluent cultures were normalized to protein levels. For measurements of VEGF in culture media, media were collected when cells were $70 \%-80 \%$ confluent and ready to be grown in adipogenic medium for 3 days for CFU-A assays. The optical density of each well was determined using a microplate reader set to $450 \mathrm{~nm}$. The optical density determined with blank culture medium was used as a control.

Immunocytochemistry. Cells seeded in culture chambers were fixed in $4 \%$ PFA and incubated with antibodies against VEGF (sc-152, Santa Cruz Biotechnology Inc.), Flt-1 (sc-316, Santa Cruz Biotechnology Inc.), Flk-1 (sc-505, Santa Cruz Biotechnology Inc.), or lamin A/C (sc-20681, Santa Cruz Biotechnology Inc.) in combination with OSX (sc-22538, Santa Cruz Biotechnology Inc.) antibodies at 1:50 dilution for 60 minutes at room temperature. After 3 washes with PBS, cells were incubated with Alexa Fluor 555-labeled donkey anti-rabbit IgG and Alexa Fluor 488-labeled donkey anti-goat IgG (Invitrogen) at 1:200 dilution for 45 minutes. DAPI was used for nuclear staining. Fluorescence images were obtained with a Nikon $80 \mathrm{i}$ Upright microscope and associated software.

Western blotting. Cell lysates were prepared by adding ice-cold PhosphoSafe Extraction Reagent (Novagen) supplemented with protease inhibitor cocktail (Roche). After centrifugation for 20 minutes $(16,500 \mathrm{~g})$ at $4^{\circ} \mathrm{C}$, protein concentrations of supernatants were determined by Bradford Protein Assay kit (Thermo Scientific). Equal amounts of protein were loaded and separated by SDS-PAGE using $4 \%-12 \%$ Bis-Tris precast polyacrylamide gels (NUPAGE; Invitrogen), followed by transfer of proteins onto nitrocellulose membranes (Bio-Rad). Blots were blocked with 5\% Blotting-Grade Blocker Nonfat Dry Milk (Bio-Rad) in TBS/0.1\% (v/v) Tween-20, followed by incubation overnight with primary antibodies against lamin $\mathrm{A} / \mathrm{C}$ (1:500, sc-20681, Santa Cruz Biotechnology Inc.), Flt-1 (1:500, sc-316, Santa Cruz Biotechnology Inc.), Flk-1 (1:500, sc-505, Santa Cruz Biotechnology Inc.), phospho-VEGFR2 (1:1,000, 2478, Cell Signaling Technology), RUNX2 (1:1,000; 8486, Cell Signaling Technology), PPAR $\gamma$ (1:1,000; 2443, Cell Signaling Technology), phospho-Akt (Ser473, 1:1,000, 9271, Cell Signaling Technology), phospho-p38 MAP Kinase (Thr180/Tyr182, 1:1,000, 9211, Cell Signaling Technology), and $\beta$-actin (1:5,000; 5441, Sigma-Aldrich) in $5 \%$ BSA in TBS $/ 0.1 \%(v / v)$ Tween-20. Following incubation with horseradish peroxidase-conjugated secondary antibodies for 1 hour, immunoreactive bands were detected by chemiluminescent substrate (Thermo Scientific).

DNA transfections and reporter assays. Bone marrow cells were plated at $2.5 \times 10^{4}$ cells per well in 24-well plates and transfected with $500 \mathrm{ng}$ OSEluc (a gift from Toshihisa Komori, Nagasaki University Graduate School of Biomedical Sciences, Nagasaki, Japan) and $12.5 \mathrm{ng}$ of a thymidine kinase promoter-driven Renilla luciferase construct (Promega) per well, using Lipofectamine LTX Plus Reagent (Invitrogen). After transfection, cells were cultured for 24 hours in osteogenic culture medium containing $100 \mu \mathrm{M}$ ascorbic acid 2-phosphate (Wako) and $8 \mathrm{mM} \beta$-glycerophosphate 
(Sigma-Aldrich). Cells were then lysed, and extracts were used in luciferase assays performed with the Dual-Glo Luciferase Assay System (Promega) in accordance with the manufacturer's instructions. Luciferase activity was measured using a microplate luminometer, and the firefly luciferase value in each well was normalized to the corresponding Renilla luciferase readout.

Blocking antibodies and recombinant proteins. Antibodies against mouse VEGF (affinity-purified polyclonal goat IgG, cat. AF-493-NA) from R\&D Systems were used for blocking experiments. The antibody concentration $(0.8 \mu \mathrm{g} / \mathrm{ml})$ in the blocking experiments with anti-VEGF is the upper limit for ELISA capture with this antibody and about 5 times higher than the upper range for the neutralization dose $\left(\mathrm{ND}_{50}\right)$ for this antibody $(0.05-0.15$ $\mu \mathrm{g} / \mathrm{ml}$ ) in the presence of $10 \mathrm{ng} / \mathrm{ml}$ recombinant VEGF164, measured by its ability to neutralize VEGF164-induced proliferation in human umbilical vein endothelial cells (65). This antibody concentration was sufficient to reduce osteoclast differentiation to the low levels seen with bone marrow cultures from $V e g f a^{f / f} ;$ Osx-Cre mice when antibody was added to cultures from Vegfal/fl mice. To test whether exogenous VEGF could rescue the changes in CFU-F assays following knockdown of Vegfa in bone marrow cells, recombinant VEGF was added at concentrations of $20 \mathrm{ng} / \mathrm{ml}, 50 \mathrm{ng} /$ $\mathrm{ml}$, and $100 \mathrm{ng} / \mathrm{ml}$. At these concentrations no rescue effects were seen. However, a concentration of $20 \mathrm{ng} / \mathrm{ml}$ of VEGF was sufficient to restore osteoclastogenesis to control levels when the protein was added to cultures of bone marrow cells from Vegfafl/f; Osx-Cre mice. Recombinant mouse VEGF (VEGF164) (cat. 493-MV/CF) was obtained from R\&D Systems.

Statistics. Results are presented as mean $\pm \mathrm{SD}$, and unpaired 2-tailed Student's $t$-test was used. $P$ values less than 0.05 were considered significant.
Study approval. All animal experiments were performed on the basis of protocols approved by the Harvard Medical Area Standing Committee on Animals and in accordance with the U.S. Public Health Service Policy on Humane Care and Use of Laboratory Animals.

\section{Acknowledgments}

We thank Hiroki Suemoto for help with isolation of bone marrow cells, Yulia Pittel for secretarial assistance, Naomi Fukai and Sofiya Plotkina for technical support, and Carl Walkly and Louise Purton for helpful discussions. We thank Nicholas Brady and Antonios Aliprantis for microCT analysis and the Nikon Imaging Center at Harvard Medical School for help with light and fluorescence microscopy. This work was supported by NIH grants AR36819, AR36820, and AR48564 (to B.R. Olsen) and partially supported by a Harvard School of Dental Medicine Dean's Scholarship (to Y. Liu). Received for publication September 28, 2011, and accepted in revised form July 5, 2012.

Address correspondence to: Bjorn R. Olsen, Harvard School of Dental Medicine, 188 Longwood Ave., Boston, Massachusetts, USA. Phone: 617.432.1874; Fax: 617.432.0638; E-mail: bjorn_ olsen@hms.harvard.edu. Or to: Yanqiu Liu, 3496 Bryant Street, Palo Alto, California 94306, USA. Phone: 617.938.8215; Fax: 650.472.2437; E-mail: yanqiu_liu@post.harvard.edu.

Shidong Jia's present address is: Genentech, South San Francisco, California, USA.
1. Rodríguez JP, Montecinos L, Ríos S, Reyes P, Martínez J. Mesenchymal stem cells from osteoporotic patients produce a type I collagen-deficient extracellular matrix favoring adipogenic differentiation. J Cell Biochem. 2000;79(4):557-565.

2. Meunier P, Aaron J, Edouard C, Vignon G. Osteoporosis and the replacement of cell populations of the marrow by adipose tissue. A quantitative study of 84 iliac bone biopsies. Clin Orthop Relat Res. 1971;80:147-154.

3. Justesen J, Stenderup K, Ebbesen EN, Mosekilde L, Steiniche T, Kassem M. Adipocyte tissue volume in bone marrow is increased with aging and in patients with osteoporosis. Biogerontology. 2001; 2(3):165-171.

4. Verma S, Rajaratnam JH, Denton J, Hoyland JA, Byers RJ. Adipocytic proportion of bone marrow is inversely related to bone formation in osteoporosis. J Clin Pathol. 2002;55(9):693-698.

5. Yeung DK, Griffith JF, Antonio GE, Lee FK, Woo $\mathrm{J}$, Leung PC. Osteoporosis is associated with increased marrow fat content and decreased marrow fat unsaturation: a proton MR spectroscopy study. J Magn Reson Imaging. 2005;22(2):279-285.

6. Rosen CJ, Bouxsein ML. Mechanisms of disease: is osteoporosis the obesity of bone? Nat Clin Pract Rheumatol. 2006;2(1):35-43.

7. Beresford JN, Bennett JH, Devlin C, Leboy PS, Owen $\mathrm{ME}$. Evidence for an inverse relationship between the differentiation of adipocytic and osteogenic cells in rat marrow stromal cell cultures. J Cell Sci. 1992;102(pt 2):341-351.

8. Nuttall ME, Gimble JM. Controlling the balance between osteoblastogenesis and adipogenesis and the consequent therapeutic implications. Curr Opin Pharmacol. 2004:4(3):290-294.

9. Pittenger MF, et al. Multilineage potential of adult human mesenchymal stem cells. Science. 1999 ; 284(5411):143-147.

10. Nuttall ME, Patton AJ, Olivera DL, Nadeau DP, Gowen M. Human trabecular bone cells are able to express both osteoblastic and adipocytic pheno- type: implications for osteopenic disorders. J Bone Miner Res. 1998;13(3):371-382.

11. Song L, Tuan RS. Transdifferentiation potential of human mesenchymal stem cells derived from bone marrow. FASEB J. 2004;18(9):980-982.

12. Ponce ML, et al. Coexpression of osteogenic and adipogenic differentiation markers in selected subpopulations of primary human mesenchymal progenitor cells. J Cell Biochem. 2008;104(4):1342-1355.

13. Bennett CN, et al. Regulation of osteoblastogenesis and bone mass by Wnt10b. Proc Natl Acad Sci U S A. 2005;102(9):3324-3329.

14. Kang S, Bennett CN, Gerin I, Rapp LA, Hankenson $\mathrm{KD}$, Macdougald OA. Wnt signaling stimulates osteoblastogenesis of mesenchymal precursors by suppressing CCAAT/enhancer-binding protein alpha and peroxisome proliferator-activated receptor gamma. J Biol Chem. 2007;282(19):14515-14524.

15. Ross SE, et al. Inhibition of adipogenesis by Wnt signaling. Science. 2000;289(5481):950-953.

16. Takada I, et al. A histone lysine methyltransferase activated by non-canonical Wnt signalling suppresses PPAR-gamma transactivation. Nat Cell Biol. 2007;9(11):1273-1285.

17. Bilkovski R, et al. Role of WNT-5a in the determination of human mesenchymal stem cells into preadipocytes. J Biol Chem. 2010;285(9):6170-6178.

18. Cui Y, et al. Lrp5 functions in bone to regulate bone mass. Nat Med. 2011;17(6):684-691.

19. Xiao Z, Zhang S, Cao L, Qiu N, David V, Quarles LD. Conditional disruption of Pkd1 in osteoblasts results in osteopenia due to direct impairment of bone formation. J Biol Chem. 2010;285(2):1177-1187.

20. Luo J, et al. TGFbeta/BMP type I receptors ALK1 and ALK2 are essential for BMP9-induced osteogenic signaling in mesenchymal stem cells. J Biol Chem. 2010;285(38):29588-29598

21. Mayr-Wohlfart U, et al. Vascular endothelial growth factor stimulates chemotactic migration of primary human osteoblasts. Bone. 2002;30(3):472-477.

22. Maes C, et al. Osteoblast precursors, but not mature osteoblasts, move into developing and frac- tured bones along with invading blood vessels. Dev Cell. 2010;19(2):329-344.

23. Hiltunen MO, et al. Adenovirus-mediated VEGFA gene transfer induces bone formation in vivo. FASEB J. 2003;17(9):1147-1149.

24. Harper J, Gerstenfeld LC, Klagsbrun M. Neuropilin-1 expression in osteogenic cells: down-regulation during differentiation of osteoblasts into osteocytes. J Cell Biochem. 2001;81(1):82-92.

25. Midy V, Plouet J. Vasculotropin/vascular endothelial growth factor induces differentiation in cultured osteoblasts. Biochem Biophys Res Commun. 1994;199(1):380-386.

26. Zelzer E, et al. Skeletal defects in VEGF(120/120) mice reveal multiple roles for VEGF in skeletogenesis. Development. 2002;129(8):1893-1904.

27. Rivard A, et al. Age-dependent impairment of angiogenesis. Circulation. 1999;99(1):111-120.

28. Pola R, et al. Age-dependent VEGF expression and intraneural neovascularization during regeneration of peripheral nerves. Neurobiol Aging. 2004; 25(10):1361-1368.

29. Wilson A, Shehadeh LA, Yu H, Webster KA. Agerelated molecular genetic changes of murine bone marrow mesenchymal stem cells. BMC Genomics. 2010;11:229.

30. Edelberg JM, et al. Platelet-derived growth factor$\mathrm{AB}$ limits the extent of myocardial infarction in a rat model: feasibility of restoring impaired angiogenic capacity in the aging heart. Circulation. 2002; 105(5):608-613.

31. Jiang S, Kh Haider H, Ahmed RP, Idris NM, Salim A, Ashraf M. Transcriptional profiling of young and old mesenchymal stem cells in response to oxygen deprivation and reparability of the infarcted myocardium. J Mol Cell Cardiol. 2008;44(3):582-596.

32. Efimenko A, Starostina E, Kalinina N, Stolzing A. Angiogenic properties of aged adipose derived mesenchymal stem cells after hypoxic conditioning. J Transl Med. 2011;9:10.

33. Swift ME, Kleinman HK, DiPietro LA. Impaired wound repair and delayed angiogenesis in aged 
mice. Lab Invest. 1999;79(12):1479-1487.

34. Nakashima K, et al. The novel zinc finger-containing transcription factor osterix is required for osteoblast differentiation and bone formation. Cell. 2002;108(1):17-29

35. Zelzer E, Mamluk R, Ferrara N, Johnson RS, Schipani E, Olsen BR. VEGFA is necessary for chondrocyte survival during bone development. Development. 2004;131(9):2161-2171.

36. Scutt A, Reading L, Scutt N, Still K. Mineralizing fibroblast-colony-forming assays. Methods Mol Med. 2003;80:29-39.

37. Niida S, et al. Vascular endothelial growth factor can substitute for macrophage colony-stimulating factor in the support of osteoclastic bone resorption. J Exp Med. 1999;190(2):293-298.

38. Niida $S$, et al. VEGF receptor 1 signaling is essential for osteoclast development and bone marrow formation in colony-stimulating factor 1-deficient mice. Proc Natl Acad Sci U S A. 2005;102(39):14016-14021.

39. Moiseeva O, Bourdeau V, Vernier M, Dabauvalle MC, Ferbeyre G. Retinoblastoma-independent regulation of cell proliferation and senescence by the p53-p21 axis in lamin A/C-depleted cells. Aging Cell. 2011;10(5):789-797.

40. Rauner M, et al. Inhibition of lamin A/C attenuates osteoblast differentiation and enhances RANKLdependent osteoclastogenesis. J Bone Miner Res. 2009;24(1):78-86.

41. Akter R, Rivas D, Geneau G, Drissi H, Duque G. Effect of lamin A/C knockdown on osteoblast differentiation and function. J Bone Miner Res. 2009; 24(2):283-293.

42. Naito M, Omoteyama K, Mikami Y, Takagi M, Takahashi T. Suppression of lamin A/C by short hairpin RNAs promotes adipocyte lineage commitment in mesenchymal progenitor cell line, ROBC26. Histochem Cell Biol. 2011;137(2):235-247.

43. Boguslavsky RL, Stewart CL, Worman HJ. Nuclear lamin A inhibits adipocyte differentiation: implications for Dunnigan-type familial partial lipodystrophy. Hum Mol Genet. 2006;15(4):653-663.
44. Li W, et al. Decreased bone formation and osteopenia in lamin a/c-deficient mice. PLoS One. 2011; 6(4):e19313.

45. Ivorra C, et al. A mechanism of AP-1 suppression through interaction of c-Fos with lamin A/C. Genes Dev. 2006;20(3):307-320.

46. Van Berlo JH, et al. A-type lamins are essential for TGF-beta 1 induced PP2A to dephosphorylate transcription factors. Hum Mol Genet. 2005; 14(19):2839-2849.

47. Naetar N, Foisner R. Lamin complexes in the nuclear interior control progenitor cell proliferation and tissue homeostasis. Cell Cycle. 2009;8(10):1488-1493.

48. Tontonoz P, Hu E, Spiegelman BM. Stimulation of adipogenesis in fibroblasts by PPAR gamma 2, a lipid-activated transcription factor. Cell. 1994; 79(7):1147-1156.

49. Costa N, Paramanathan S, Mac Donald D, Wierzbicki AS, Hampson G. Factors regulating circulating vascular endothelial growth factor (VEGF): association with bone mineral density (BMD) in post-menopausal osteoporosis. Cytokine. 2009; 46(3):376-381.

50. Gerber HP, et al. VEGF regulates haematopoietic stem cell survival by an internal autocrine loop mechanism. Nature. 2002;417(6892):954-958.

51. Lee $S$, et al. Autocrine VEGF signaling is required for vascular homeostasis. Cell. 2007;130(4):691-703

52. Samuel S, Fan F, Dang LH, Xia L, Gaur P, Ellis LM. Intracrine vascular endothelial growth factor signaling in survival and chemoresistance of human colorectal cancer cells. Oncogene. 2011;30(10):1205-1212.

53. Lee T-H, et al. Vascular endothelial growth factor mediates intracrine survival in human breast carcinoma cells through internaly expressed VEGFR1/ FLT1. PLoS Med. 2007;4(6):e186.

54. Li W, Keller G. VEGF nuclear accumulation correlates with phenotypical changes in endothelial cells. J Cell Sci. 2000;113(pt 9):1525-1534.

55. Domingues I, Rino J, Demmers JA, de Lanerolle P, Santos SC. VEGFR2 translocates to the nucleus to regulate its own transcription. PLoS One. 2011; 6(9):e25668.

56. Zhang Y, et al. Co-stimulation of the bone-related Runx2 P1 promoter in mesenchymal cells by SP1 and ETS transcription factors at polymorphic purine-rich DNA sequences (Y-repeats). J Biol Chem. 2009;284(5):3125-3135.

57. Kwon TG, et al. Physical and functional interactions between Runx2 and HIF-1alpha induce vascular endothelial growth factor gene expression. J Cell Biochem. 2011;112(12):3582-3593.

58. Schilling T, Kuffner R, Klein-Hitpass L, Zimmer R, Jakob F, Schutze N. Microarray analyses of transdifferentiated mesenchymal stem cells. J Cell Biochem. 2008;103(2):413-433.

59. Wang Y, et al. The hypoxia-inducible factor alpha pathway couples angiogenesis to osteogenesis during skeletal development. J Clin Invest. 2007; 117(6):1616-1626.

60. Guillotin B, Bareille R, Bourget C, Bordenave L, Amedee J. Interaction between human umbilical vein endothelial cells and human osteoprogenitors triggers pleiotropic effect that may support osteoblastic function. Bone. 2008;42(6):1080-1091.

61. Gerber HP, et al. VEGF is required for growth and survival in neonatal mice. Development. 1999; 126(6):1149-1159.

62. Rodda SJ, McMahon AP. Distinct roles for Hedgehog and canonical Wnt signaling in specification, differentiation and maintenance of osteoblast progenitors. Development. 2006;133(16):3231-3244.

63. Parfitt AM, et al. Bone histomorphometry: standardization of nomenclature, symbols, and units. Report of the ASBMR Histomorphometry Nomenclature Committee. J Bone Miner Res. 1987;2(6):595-610.

64. Bakker A, Klein-Nulend J. Osteoblast isolation from murine calvariae and long bones. Methods Mol Med. 2003;80:19-28.

65. Conn G, Soderman DD, Schaeffer MT, Wile M, Hatcher VB, Thomas KA. Purification of a glycoprotein vascular endothelial cell mitogen from a rat glioma-derived cell line. Proc Natl Acad Sci U S A. 1990;87(4):1323-1327. 